\title{
Radio observations of supernova remnant G1.9+0.3
}

\section{Kieran J. Luken ${ }^{\oplus},{ }^{1,2 \star}$ Miroslav D. Filipović, ${ }^{1}$ Nigel I. Maxted ${ }^{\oplus}, 1,3$ Roland Kothes, ${ }^{4}$} Ray P. Norris ${ }^{\oplus}, 1,2$ James R. Allison ${ }^{\odot}, 5$ Rebecca Blackwell, ${ }^{6}$ Catherine Braiding,,${ }^{6,7}$ Robert Brose,${ }^{8,9}$ Michael Burton, ${ }^{10}$ Ain Y. De Horta, ${ }^{1}$ Tim J. Galvin ${ }^{\odot}, 1,11$ Lisa Harvey-Smith ${ }^{\odot},{ }^{1,7}$ Natasha Hurley-Walker, ${ }^{12}$ Denis Leahy ${ }^{\odot}, 13$ Nicholas O. Ralph, ${ }^{1}$ Quentin Roper, ${ }^{1}$ Gavin Rowell, ${ }^{6}$ Iurii Sushch, $, 9,14,15$ Dejan Urošević ${ }^{16,17}$ and Graeme F. Wong ${ }^{1,7,18}$

${ }^{1}$ Western Sydney University, Locked Bag 1797, Penrith, NSW 2751, Australia

${ }^{2}$ CSIRO Astronomy and Space Sciences, Australia Telescope National Facility, P.O. Box 76, Epping, NSW 1710, Australia

${ }^{3}$ School of Science, The University of New South Wales, Australian Defence Force Academy, Canberra, ACT 2600, Australia

${ }^{4}$ Dominion Radio Astrophysical Observatory, Herzberg Programs in Astronomy and Astrophysics, National Research Council Canada, P.O. Box 248, Penticton, BC V2A 6J9, Canada

${ }^{5}$ Sub-Department of Astrophysics, Department of Physics, University of Oxford, Denys Wilkinson Building, Keble Rd., Oxford OX1 3RH, UK

${ }^{6}$ School of Physical Sciences, The University of Adelaide, Adelaide 5005, Australia

${ }^{7}$ School of Physics, The University of New South Wales, Sydney 2052, Australia

${ }^{8}$ DESY, D-15738 Zeuthen, Germany

${ }^{9}$ Institute of Physics and Astronomy, University of Potsdam, D-14476 Potsdam, Germany

${ }^{10}$ Armagh Observatory and Planetarium, College Hill, Armagh, BT61 9DG, Northern Ireland, UK

${ }^{11}$ CSIRO Astronomy and Space Science, P.O. Box 1130, Bentley WA 6102, Australia

${ }^{12}$ International Centre for Radio Astronomy Research, Curtin University, Bentley, WA 6102, Australia

${ }^{13}$ Department of Physics and Astronomy, University of Calgary, University of Calgary, Calgary, Alberta T2N 1 N4, Canada

${ }^{14}$ Centre for Space Research, North-West University, 2520 Potcheftroom, South Africa

${ }^{15}$ Astronomical Observatory of Ivan Franko National University of L'viv, vul. Kyryla i Methodia, 8, L'viv 79005, Ukraine

${ }^{16}$ Department of Astronomy, Faculty of Mathematics, University of Belgrade, Studentski trg 16, 11000 Belgrade, Serbia

${ }^{17}$ Isaac Newton Institute of Chile, Yugoslavia Branch

${ }^{18}$ Pawsey Supercomputing Centre, 26 Dick Perry Ave, Kensington, WA 6151, Australia

Accepted 2019 December 3. Received 2019 December 2; in original form 2019 July 30

\begin{abstract}
We present $1-10 \mathrm{GHz}$ radio continuum flux density, spectral index, polarization, and rotation measure (RM) images of the youngest known Galactic supernova remnant (SNR) G1.9+0.3, using observations from the Australia Telescope Compact Array. We have conducted an expansion study spanning eight epochs between 1984 and 2017, yielding results consistent with previous expansion studies of G1.9+0.3. We find a mean radio continuum expansion rate of $(0.78 \pm 0.09)$ per cent $\mathrm{yr}^{-1}$ (or $\sim 8900 \mathrm{~km} \mathrm{~s}^{-1}$ at an assumed distance of $8.5 \mathrm{kpc}$ ), although the expansion rate varies across the SNR perimetre. In the case of the most recent epoch between 2016 and 2017, we observe faster-than-expected expansion of the northern region. We find a global spectral index for G1.9+0.3 of $-0.81 \pm 0.02(76 \mathrm{MHz}-10 \mathrm{GHz})$. Towards the northern region, however, the radio spectrum is observed to steepen significantly $(\sim-1)$. Towards the two so-called (east and west) 'ears' of G1.9+0.3, we find very different RM values of 400-600 and 100-200 $\mathrm{rad} \mathrm{m}^{2}$, respectively. The fractional polarization of the radio continuum emission reaches $(19 \pm 2)$ per cent, consistent with other, slightly older, SNRs such as Cas A.
\end{abstract}

Key words: supernovae: general - ISM: individual objects: G1.9+0.3 - ISM: supernova remnants - radio continuum: ISM. 


\section{INTRODUCTION}

There are currently only $\sim 10$ confirmed 'young' (defined as being less than 2000 years old) Galactic supernova remnants (SNRs) out of a predicted $~ 50$ (van den Bergh \& Tammann 1991; Cappellaro, Barbon \& Turatto 2005). The SNR G1.9+0.3 is believed to be the youngest in the Milky Way (MW) with an age (calculated from its expansion rate) of $\sim 150 \mathrm{yr}$ (Borkowski et al. 2017; De Horta et al. 2014; Reynolds et al. 2009, 2008; Green et al. 2008; Carlton et al. 2011). Pavlović (2017) estimated the age of G1.9+0.3 as $120 \mathrm{yr}$, based on an analysis of the hydrodynamical and radio evolution of this young SNR. Previous studies suggest G1.9+0.3 is a Type Ia SNR (Borkowski et al. 2013). The high expansion velocity of $\mathrm{G} 1.9+0.3$, absence of an obvious pulsar wind nebulae (PWNe), and bilateral symmetry of the X-ray emission have all been previously used as evidence (Borkowski et al. 2017). Borkowski et al. (2013) further postulated that only a very unusual core-collapse event could reproduce the observations, while a reasonable supernova (SN) Type Ia model can reach the observed size and velocity with a mean external density of $\sim 0.02 \mathrm{~cm}^{3}$ (Borkowski et al. 2010). Therefore, detailed studies of this fast evolving SNR will give us unprecedented insight into the evolution of SNRs in general, with a particular interest in the early stages of their evolution, the dynamics of SN ejecta and on particle acceleration.

Upon its discovery with the Very Large Array (VLA; Green \& Gull 1984), G1.9+0.3 was noted to have a radio brightness comparable to the Tycho and Kepler SNRs with a spectral index of $\alpha \sim-0.6 .{ }^{1}$ Molonglo Observatory Synthesis Telescope (MOST) Galactic Survey data resolved a shell-like morphology with diameter 1.2 arcmin (Gray 1994). Subsequent studies of 20/90 cm VLA data characterized the SNR as having diameter $\lesssim 1$ arcmin and spectral index of $\alpha=-0.93 \pm 0.23$ (LaRosa et al. 2000; Nord et al. 2004), with the steep spectral index suggesting the radio emission is primarily synchrotron based. Farnes (2012) later mapped the spatial variation in polarization and spectral index, noting flatter spectra in the NW and SE of the remnant.

Distance is a key variable for calculating expansion velocity and age. Nord et al. (2004) inferred the distance to be $<7.8 \mathrm{kpc}$ using the lack of $74 \mathrm{MHz}$ absorption by the Galactic Plane as an indicator that G1.9+0.3 is on the near side of the Galactic Centre (GC). This distance is consistent with X-ray absorption studies (Green et al. 2008). Subsequently, H I absorption in the so-called FeatureI gas structure (Cohen 1975, See Section 3.1) was observed in the $\mathrm{G} 1.9+0.3$ radio continuum emission, implying that the SNR is beyond Feature-I (Roy \& Pal 2014). Feature-I extends $>5^{\circ}$ in Galactic longitude and does not appear in $\mathrm{HI}$-absorption towards the Sagittarius A* radio continuum, so this component lies beyond the precise GC distance. It follows that G1.9+0.3, as it corresponds with Feature-I, must also lie beyond the GC, which, following IAU standards, we assume to lie at a distance of $8.5 \mathrm{kpc}$ (Kerr \& LyndenBell 1986). However, we do note the recent disagreement in this fundamental parameter by Francis \& Anderson (2014, $7.4 \mathrm{kpc}$ ), and de Grijs \& Bono $(2016, \sim 8.3 \mathrm{kpc})$. The far $3 \mathrm{kpc}$ Expanding arm at line of sight velocity $\sim+50 \mathrm{~km} \mathrm{~s}^{-1}$, does not appear in absorption (Roy \& Pal 2014), therefore G1.9+0.3 is in front of this component. We therefore assume G1.9+0.3 to have a distance of $8.5 \mathrm{kpc}$, which is consistent with both X-ray absorption studies (Carlton et al. 2011), and the lower and upper distance constraints derived from Feature-I and the far Expanding arm, respectively.

\footnotetext{
${ }^{1}$ Spectral index is defined as $S \propto v^{\alpha}$
}

Green et al. (2008) re-observed G1.9+0.3 at $4.86 \mathrm{GHz}$ using the VLA after Reynolds et al. (2008) used 2007 Chandra images to show G1.9+0.3 had expanded significantly since 1985 and its X-ray emission appeared to be predominantly synchrotron in nature. By comparing these new VLA observations with the 1985 VLA observations made at $1.49 \mathrm{GHz}$, Green et al. (2008) determined that G1.9+0.3 had expanded by $15 \pm 2$ percent over 23 yr $\left(\sim 0.65\right.$ percent $\left.\mathrm{yr}^{-1}\right)$. Using only the 1985 and 1989 VLA observations, Gómez \& Rodríguez (2009) derived a smaller expansion rate of $0.46 \pm 0.11$ percent, and estimated the G1.9+0.3 age to be $220_{-45}^{+70}$ yr. De Horta et al. (2014) later used all available ATCA (Australia Telescope Compact Array) and VLA radio-continuum observations at $6 \mathrm{~cm}$, to estimate a median expansion rate of $0.563 \pm 0.078$ percent $\mathrm{yr}^{-1}$ between 1984 and 2009. It was noted that the apparent expansion of G1.9+0.3 was slower $\left(0.484\right.$ percent $\left.\mathrm{yr}^{-1}\right)$ in the 1980 s compared to recent epochs $\left(2014 ; 0.641\right.$ per cent $\left.\mathrm{yr}^{-1}\right)$.

X-ray observations have also been used to measure expansion, with Carlton et al. (2011) finding an expansion rate of $0.642 \pm 0.049$ percent $\mathrm{yr}^{-1}$ and a flux density increase of $1.7 \pm 1.0$ percent $\mathrm{yr}^{-1}$ by comparing 2007 and 2009 Chandra images. A simple uniform-expansion model leads to a $\mathrm{G} 1.9+0.3$ age estimate of $156 \pm 11 \mathrm{yr}$ old assuming no deceleration, however, a uniform expansion model is probably an oversimplification. Borkowski et al. (2013) recorded ejecta from G1.9+0.3 to have speeds as large as $\sim 18000 \mathrm{~km} \mathrm{~s}^{-1}$. In their study, an implied abundance inhomogeneity of Fe-rich northern ejecta and Si/S-rich eastern ejecta were said to be consistent with asymmetrical Type Ia SN explosion models.

As G1.9+0.3 expanded, it also brightened. Murphy, Gaensler \& Chatterjee (2008) found that the flux density of G1.9+0.3 at $843 \mathrm{MHz}$ increased by $\left(1.22 \pm_{0.16}^{0.24}\right)$ percent $\mathrm{yr}^{-1}$ over the last two decades. From simulations based on the non-linear diffuse shock acceleration (NLDSA) model, Pavlović (2017) found that the radio flux density should have increased by $\sim 1.8$ per cent $\mathrm{yr}^{-1}$ over the past two decades. Such behaviour would be consistent with an SNR sweeping up the surrounding interstellar medium (ISM) increasing number of particles which can be injected into the NLDSA process, gaining ultrarelativistic energies and emitting synchrotron radiation. Additionally, this numerical model predicts that the radio flux density will increase, reaching its maximum value around $500 \mathrm{yr}$ from now. During the late-free expansion phase, the SNR flux density will start to decrease. The beginning of Sedov phase will start around $1700 \mathrm{yr}$ after the initial SN explosion. Pavlović (2017) emphasized that in this stage of evolution of G1.9+0.3, we are witnessing the fastest increase in radio brightness this SNR will ever produce. Moreover the steep radio continuum spectrum of G1.9+0.3 obtained from various observations can be explained as a result of the efficient NLDSA accompanying with strong magnetic field amplification (Pavlović 2017).

However, Reynolds et al. (2008) noted that the radii of the X-ray and radio shells of G1.9+0.3 differ by $\approx 20$ per cent. This difference cannot be explained if electrons are accelerated only at the forward shock of G1.9+0.3 as the maximum offset possible in the case of very efficient magnetic field amplification and thus efficient synchrotron cooling is of the order of 5 per cent. Brose et al. (2019) showed that the different expansion and brightening of the radio and X-ray shells can be explained if the X-ray emission originates at the forward shock and the radio emission mainly at the reverse shock. Efficient particle acceleration at the reverse shock has been proposed earlier (Telezhinsky, Dwarkadas \& Pohl 2013). Combined with the young age of G1.9+0.3, a reverse-shock density three times 
Table 1. 2016 and 2017 ATCA observation details of G1.9+0.3. This table includes the date of the observations, the array configuration, the number of channels, the bandwidth in $\mathrm{MHz}$, and the frequency in $\mathrm{MHz}$.

\begin{tabular}{lcccc}
\hline Date & $\begin{array}{c}\text { Array } \\
\text { configuration }\end{array}$ & Channels & $\begin{array}{c}\text { Bandwidth } \\
(\mathrm{MHz})\end{array}$ & $\begin{array}{c}\text { Frequency } v \\
(\mathrm{MHz})\end{array}$ \\
\hline 2016 January 26-27 & EW352 & 5121 & 2.5 & 1421 \\
2016 January 26-27 & EW352 & 2049 & 1 & $1610,1664,1666,1719$ \\
2016 January 26-27 & EW352 & 2049 & 2048 & $2100,5000,9000$ \\
2016 March 8-9 & 6B & 2049 & 2048 & $2100,5000,9000$ \\
2017 May 20-21 & 6A & 5121 & 2.5 & 1421 \\
2017 May 20-21 & 6A & 2049 & 1 & $1610,1664,1666,1719$ \\
2017 May 20-21 & 6A & 2049 & 2048 & $2100,5000,9000$ \\
\hline
\end{tabular}

higher than the forward-shock density and a reverse shock speed of $\approx 5600 \mathrm{~km} \mathrm{~s}^{-1}$ in the plasma frame, $\mathrm{G} 1.9+0.3$ is a prime candidate for the detectable non-thermal emission from the reverse shock.

G1.9+0.3 has previously been observed with the ATCA. However, the Compact Array Broadband Backend upgrade (Wilson et al. 2011) increased the resolution, sensitivity, and bandwidth of the array. This allows us a detailed look at the SNR morphology and spectrum, as well as providing additional epochs with which to measure the expansion of this young Galactic SNR. Observations of G1.9+0.3 from 1984, 1985, 1987, 1989, and 2008 (taken from the VLA archives) and our ATCA observations (from 2009, 2016 and 2017) give us an opportunity to conduct a high-precision study of its expansion in the radio regime.

In this paper, we present the results from 2016 and 2017 ATCA observations of G1.9+0.3. In Section 3.1, we investigate $\mathrm{HI}$ absorption and $\mathrm{CO}(1-0)$ structure towards $\mathrm{G} 1.9+0.3$ in the context of previous studies. We derive the expansion rate of $\mathrm{G} 1.9+0.3$ using the new observations taken using the ATCA and previous images from the ATCA and VLA in Section 3.2. This is followed by our polarization and rotation measure (RM) studies of the SNR in Section 3.3. In Section 3.4, we present spectral index maps, and calculate a revised G1.9+0.3 spectral index using our ATCA observations and data from the GaLactic and Extragalactic All-sky MWA survey (GLEAM) project (Wayth et al. 2015). In Section 3.5, we then calculate the general and local radio flux density increase of $\mathrm{G} 1.9+0.3$.

The results are discussed in Section 4, with the conclusion presented in Section 5.

\section{DATA AND OBSERVATIONS}

We observed the SNR G1.9+0.3 in 2016 and 2017 using the ATCA across three $12 \mathrm{~h}$ periods (Project C1952; Table 1). Using the EW352 array on 2016 January 26-27, we observed in spectral line (observed at $1421,1610,1664,1666$, and $1719 \mathrm{MHz}$ ) and continuum mode (2100, 5000, and $9000 \mathrm{MHz}$ ) using a frequency switching technique. The 6B array was used on 2016 March 8-9 in continuum mode (2.1, 5, and $9 \mathrm{GHz})$. On 2017 May 20-21, we used the 6A array in spectral line mode (observing at 1421, 1610, 1664, 1666, and $1719 \mathrm{MHz})$ and continuum mode $(2100,5000$, and $9000 \mathrm{MHz})$ using a frequency switching mode. Over this time and across all arrays used, there was a total of 44 unique baselines, covering a range of spacings between 30 and $5969 \mathrm{~m}$ and giving us excellent $u v$ coverage.

The same flux density calibrator (1934-628) and phase calibrator (1710-269) were used for all observations. The complete observational details are available in Table 1.

The MIRIAD (Sault, Teuben \& Wright 1995) software package was used to reduce the data in multifrequency synthesis mode, with the deconvolution being completed with the INVERT, MFCLEAN, and RESTOR tasks, and primary beam correction with the LINMOS task with shadowed data flagged out. Figs 1 and 2 show the final continuum images at each of observed frequencies $(2.1,5$, and $9 \mathrm{GHz}$ ), as well as combined (2.1 and 5, and 2.1,5, and $9 \mathrm{GHz}$ ), with the detailsof these images in Table 2. The two combination images were synthesized using a restricted $u v$ range within the INVERT task to ensure appropriate (common) $u v$ coverage. These images are the most sensitive (rms noise of $0.05 / 0.06 \mathrm{mJy}^{\text {beam }}{ }^{-1}$ ) and prove to be ideal for our expansion study (Section 3.2), and localized spectral index study (Section 3.4). However, we acknowledge that the physical meaning of such images is ambiguous, since different emission mechanisms are contributing emission on different scales at different frequencies.

The 2016 images at 2.1 and $5 \mathrm{GHz}$ were created using a ROBUST of -1 , the $9 \mathrm{GHz}$ image using a ROBUST ('Briggs Weighting') of 0 , and the two combination images with a ROBUST of -1 . All images were phase self-calibrated using the SELFCAL task. The 2017 images are all prepared using a ROBUST of -1 . Images in Figs 1 and 2 were produced using the CGDISP task, and analysed using the KARMA software package (Gooch 2011).

The H I absorption study in Section 3.1 was completed using the Stokes $I$ image cube produced by MIRIAD with its INVERT, CLEAN, RESTOR, UVMODEL, UVLIN - subtracting continuum using a linear model based on 1900 line-free channels - tasks details of the images used are in Table 3.

The expansion study shown in Section 3.2 was completed using the Stokes $I$ images produced by MIRIAD with its INVERT, MFCLEAN, RESTOR, LINMOS tasks and shell profiles measured using the CGSLICE task. Figs 5-7 were created using the MATPLOTLIB PYTHON library (Hunter 2007).

The polarization study in Section 3.3 was completed using the Stokes $I, Q$, and $U$ images at $5 \mathrm{GHz}$ produced by MIRIAD with its INVERT, MFCLEAN, RESTOR, and IMPOL tasks. The images in Fig. 8 were produced by smoothing to a common resolution of 9 arcsec and then overlaying the polarization vectors on a continuum image using the CGDISP task. The RM in this section was measured by splitting the $2 \mathrm{GHz}$ bandwidth into four equal bands.

The spectral index and spectral index map in Section 3.4 were completed using the Stokes $I$ images produced by MIRIAD with its INVERT, MFCLEAN, RESTOR, LINMOS, and MFSPIN tasks. Fig. 11 was created using the CGDISP task. The spectral index map is created using a combined $2.1,5$, and $9 \mathrm{GHz}$ image, with the $u v$ coverage tapered to ensure that all frequencies cover the same $u v$ range. This resulted in better sensitivity over the wider frequency range at the expense of somewhat lower resolution.

To complement our ATCA data, we examined preliminary ${ }^{12} \mathrm{CO}(1-0)$ and ${ }^{13} \mathrm{CO}(1-0)$ data from the Mopra Southern Galactic 
Table 2. 2016 and 2017 ATCA image details of G1.9+0.3. Includes the year of the observations, the frequency in $\mathrm{GHz}$, the rms noise in $\mathrm{mJy}_{\text {beam }}{ }^{-1}$, the synthesized beam size and position angle, and the robust weighting scheme used during imaging (where lower values minimize sidelobes, and larger values optimize signal to thermal noise).

\begin{tabular}{|c|c|c|c|c|c|}
\hline Year & $\begin{array}{l}\text { Frequency } v \\
\quad(\mathrm{GHz})\end{array}$ & $\begin{array}{c}\text { Rms noise } \\
\left(\mathrm{mJy}^{2} \text { beam }^{-1}\right)\end{array}$ & $\begin{array}{l}\text { Synthesized } \\
\text { beam }\end{array}$ & $\begin{array}{c}\text { Position } \\
\text { angle }\end{array}$ & Robust \\
\hline 2016 & 2.1 & 0.03 & $\begin{array}{c}6.04 \operatorname{arcsec} \times \\
2.41 \operatorname{arcsec}\end{array}$ & $-0.8^{\circ}$ & -1 \\
\hline 2016 & 5.0 & 0.10 & $\begin{array}{c}2.64 \operatorname{arcsec} \times \\
1.15 \operatorname{arcsec}\end{array}$ & $-5.1^{\circ}$ & -1 \\
\hline 2016 & 9.0 & 0.11 & $\begin{array}{c}2.28 \operatorname{arcsec} \times \\
0.98 \operatorname{arcsec}\end{array}$ & $-6.2^{\circ}$ & 0 \\
\hline 2016 & $2.1+5.0$ & 0.06 & $\begin{array}{c}3.63 \operatorname{arcsec} \times \\
2.12 \operatorname{arcsec}\end{array}$ & $-4.1^{\circ}$ & -1 \\
\hline 2016 & $2.0+5.0+9.0$ & 0.05 & $\begin{array}{c}3.49 \operatorname{arcsec} \times \\
2.11 \operatorname{arcsec}\end{array}$ & $-5.1^{\circ}$ & -1 \\
\hline 2017 & 2.1 & 0.12 & $\begin{array}{c}5.35 \operatorname{arcsec} \times \\
2.06 \operatorname{arcsec}\end{array}$ & $+2.4^{\circ}$ & -1 \\
\hline 2017 & 5.0 & 0.13 & $\begin{array}{c}2.81 \operatorname{arcsec} \times \\
1.01 \operatorname{arcsec}\end{array}$ & $+3.2^{\circ}$ & -1 \\
\hline
\end{tabular}

Plane CO Survey (Burton et al. 2013), taken between 2013 and 2018 by the 22-m Mopra radio telescope, located in the Warrambungles National Park, Australia. The full survey data release will cover longitudes of $-110^{\circ}<l<11^{\circ}$ and latitudes of $|b|<1^{\circ}$, with extensions in selected regions of interest (Braiding et al. 2018). The full survey also includes the $\mathrm{C}^{18} \mathrm{O}(1-0)$ and $\mathrm{C}^{17} \mathrm{O}(1-0)$ isotopologue transitions, however these were not available for this investigation. The specific central molecular zone (CMZ) data presented in this paper are preliminary and will be publicly released by Blackwell et al. (In Prep.), who also outline the full data reduction process.

Mopra CO data have a 36 arcsec angular resolution. The Mopra spectrometer, MOPS, has eight 4096-channel dual-polarization bands that deliver spectra with a velocity resolution of $0.1 \mathrm{~km} \mathrm{~s}^{-1}$ when in 'zoom'-mode. The full velocity range of the $\mathrm{CO}$ data scrutinized in our analysis is $\left|v_{\mathrm{LSR}}\right|<300 \mathrm{~km} \mathrm{~s}^{-1}$, encompassing all of the known molecular components within the CMZ.

\section{RESULTS AND ANALYSIS}

\subsection{Absorption}

Fig. 3 displays spectra for Mopra ${ }^{12} \mathrm{CO}(1-0),{ }^{13} \mathrm{CO}(1-0)$, and ATCA $\mathrm{H}$ I towards $\mathrm{G} 1.9+0.3$. Both a raw $\mathrm{H}$ I spectrum and a residual $\mathrm{HI}$ spectrum, where the H I spectrum from a neighbouring region is subtracted, are shown. Absorption previously observed by Roy \& $\mathrm{Pal}$ (2014) at $\sim 10 \mathrm{~km} \mathrm{~s}^{-1}$ is visible in our residual $\mathrm{H}$ I spectrum.

Roy \& Pal (2014) attributed this gas component to local and Sagittarius arm gas. ${ }^{12} \mathrm{CO}(1-0)$ and ${ }^{13} \mathrm{CO}(1-0)$ emission indicates that molecular gas is also present towards G1.9+0.3 at a line-ofsight velocity $\sim 10 \mathrm{~km} \mathrm{~s}^{-1}$ and it may be associated with an atomic component corresponding to the H I-dip present in the residual $\mathrm{H} \mathrm{I}$ spectrum. However, since the central line velocities are offset by $\sim 3 \mathrm{~km} \mathrm{~s}^{-1}\left(\sim 7 \mathrm{~km} \mathrm{~s}^{-1}\right.$ for $\mathrm{CO}$ and $\sim 10 \mathrm{~km} \mathrm{~s}^{-1}$ for $\mathrm{HI}$ ), we make no firm conclusion regarding an association. Additionally, the complex nature of the GC makes it difficult to disentangle the foreground and background sources

Roy \& Pal (2014) also found H I absorption components at -50 and $+150 \mathrm{~km} \mathrm{~s}^{-1}$. Our ATCA residual H I spectrum does not clearly show either feature, so we do not make any new firm conclusions regarding the foreground/background nature of line of sight $\mathrm{HI}$ components, and assume a distance of $\sim 8.5 \mathrm{kpc}$ in our analysis.

Fig. 4 is a position-velocity image of Mopra $\mathrm{CO}(1-0)$ emission towards $\sim 0.9^{\circ}$ of longitude encompassing G1.9+0.3. This image shows near-zero and $\sim 50 \mathrm{~km} \mathrm{~s}^{-1}$ components visible in the $\mathrm{CO}(1-0)$ spectrum (Fig. 3), as well as gas at $+150 \mathrm{~km} \mathrm{~s}^{-1}$ that can not be discerned in Fig. 3. As noted by Roy \& Pal (2014), gas at $+150 \mathrm{~km} \mathrm{~s}^{-1}$ likely corresponds to the so-called Feature-I, which is close to the inner GC and extends to be background to Sgr A*. As noted earlier, this feature is seen in H I absorption by Roy \& Pal (2014), but not confirmed in our analysis of ATCA H I data. We further note that a tentative $\mathrm{H} \mathrm{I}$ emission component at $+150 \mathrm{~km} \mathrm{~s}^{-1}$ may exist in Fig. 3. However, since 'Feature-I' is very close to the GC, a confirmation of this component would have little effect on the assumed G1.9+0.3 distance of $8.5 \mathrm{kpc}$.

\subsection{Expansion}

Given the lack of known young Galactic SNRs, confirmation of the age as well as the measurement of its expansion is very important for evolutionary studies. The calculation of G1.9+0.3 age and expansion rate follows the method described in De Horta et al. (2014) and Roper et al. (2018)

Expansion is calculated across multiple radio-continuum images from between 1984 and 2017, produced from observations using both the VLA and ATCA. Details of the observations are in Table 4 . These images were smoothed/convolved to a single beam size (matched to the lowest resolution image $-11.12 \operatorname{arcsec} \times$ $5.32 \mathrm{arcsec}$ ). They were then regridded to ensure that all images had the same centre of RA(J2000) $=17^{h} 48^{m} 45^{s}$ and Dec. $(\mathrm{J} 2000)=-$ $27^{\circ} 10^{\prime} 6.7^{\prime \prime}$ and same pixel size. Shell profiles were then measured over 32 arcs, beginning at due west and continuing counterclockwise (paralactic angle, see Fig. 6). 32 arcs were chosen to avoid oversampling the images, giving a shell profile every $\sim 11^{\circ}$ in SNR shell azimuth, demonstrated in Fig. 7.

Using the shell profiles measured from all eight epochs over a single arc, we measure the distance from the SNR centre to the peak radio brightness along the shell. These radii data points were plotted against corresponding years, and a least-squares fit to the line was used to determine the expansion rate in arcseconds per unit 

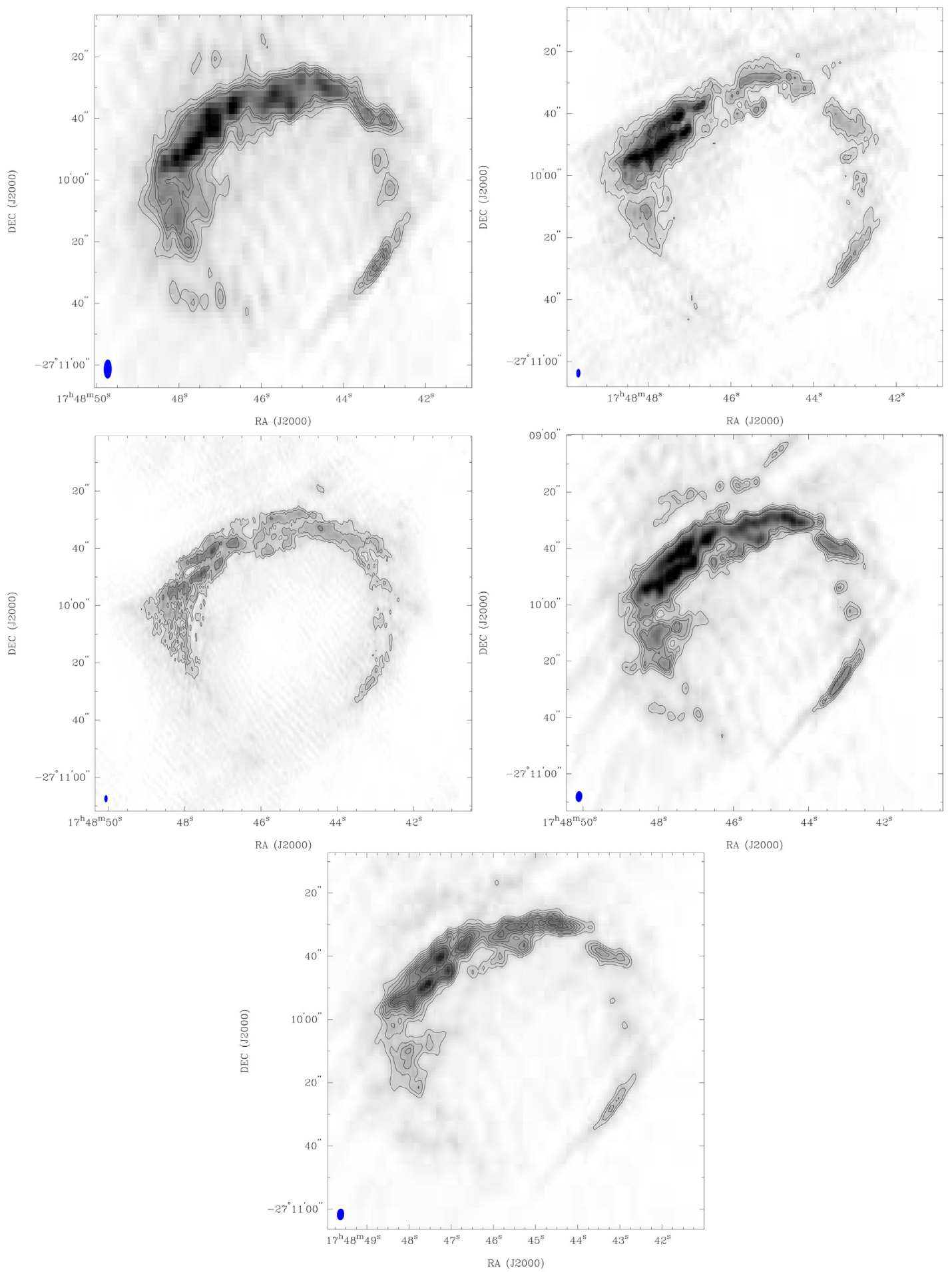

Figure 1. Radio images produced from the 2016 observations of G1.9+0.3 using the ATCA. From top left to bottom: $2.1,5,9,2.1$, and $5 \mathrm{GHz}$ combined and 2.1, 5, and $9 \mathrm{GHz}$ combined. The ellipse in the lower left corner of each is representative of the beam size (details in Table 2). Contour levels for the $2.1 \mathrm{GHz}$ image are $15 \sigma, 20 \sigma, 25 \sigma, 30 \sigma$, and $33 \sigma\left(\sigma=0.16 \mathrm{mJy}_{\text {beam }}{ }^{-1}\right) .5 .0 \mathrm{GHz}$ contours are $2 \sigma, 3 \sigma, 5 \sigma$, and $10 \sigma\left(\sigma=0.10 \mathrm{mJy}^{\mathrm{beam}}{ }^{-1}\right) .9 .0 \mathrm{GHz}$ contours are $2 \sigma, 3 \sigma, 5 \sigma$, and $10 \sigma\left(\sigma=0.11 \mathrm{mJy}_{\text {beam }}^{-1}\right) .2 .1$ and $5.0 \mathrm{GHz}$ combined image contours are $15 \sigma, 20 \sigma, 25 \sigma, 30 \sigma$, and $33 \sigma\left(\sigma=0.06 \mathrm{mJy}^{\mathrm{beam}}{ }^{-1}\right) .2 .1,5.0$, and $9.0 \mathrm{GHz}$ combined image contours are $3 \sigma, 4 \sigma, 5 \sigma, 6 \sigma, 7 \sigma, 8 \sigma, 9 \sigma$, and $10 \sigma\left(\sigma=0.05 \mathrm{mJy}^{\text {beam }}{ }^{-1}\right)$.

of time. The residuals from the fitted line were used to establish the statistical uncertainty of this expansion rate. In this analysis, the southern breakout region where no clear shell profile could be discerned was excluded, demonstrated in Fig. 7.
Once the entire SNR (at each given observation date) had been measured and fitted lines produced, we estimated the expansion of G1.9+0.3 (in arcseconds per year, percentage per year, and kilometres per second), and finally, the approximate free-expansion 

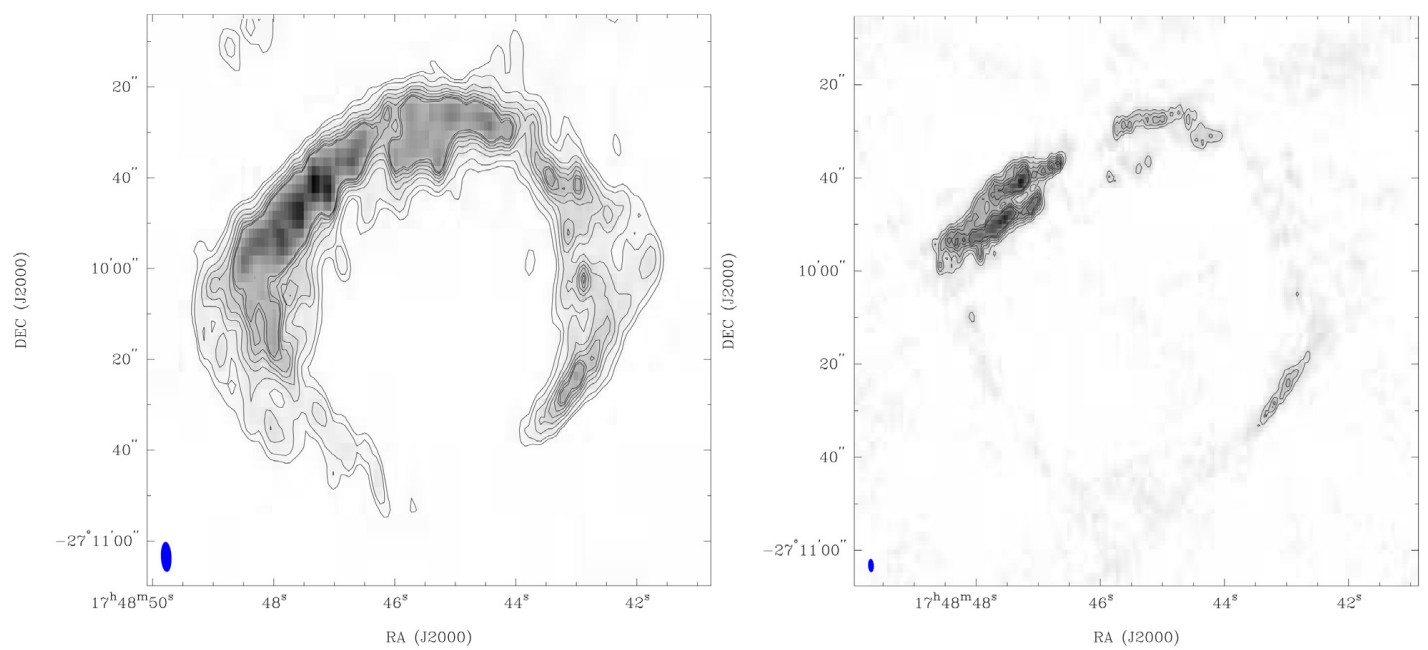

Figure 2. Radio images produced from the 2017 observations of G1.9+0.3 using the ATCA. The left-hand image is $2.1 \mathrm{GHz}$, with the right being $5 \mathrm{GHz}$. The ellipse in the lower left corner of each is representative of the beam size (details in Table 2). Contour levels for the $2.1 \mathrm{GHz}$ image are $3 \sigma, 5 \sigma, 10 \sigma, 15 \sigma, 20 \sigma$, $25 \sigma, 30 \sigma$, and $33 \sigma\left(\sigma=0.12 \mathrm{mJy}_{\text {beam }}^{-1}\right) .5 .0 \mathrm{GHz}$ contours are $2 \sigma, 3 \sigma, 5 \sigma, 6 \sigma$, and $7 \sigma\left(\sigma=0.13 \mathrm{mJy}^{\text {beam }}{ }^{-1}\right)$.

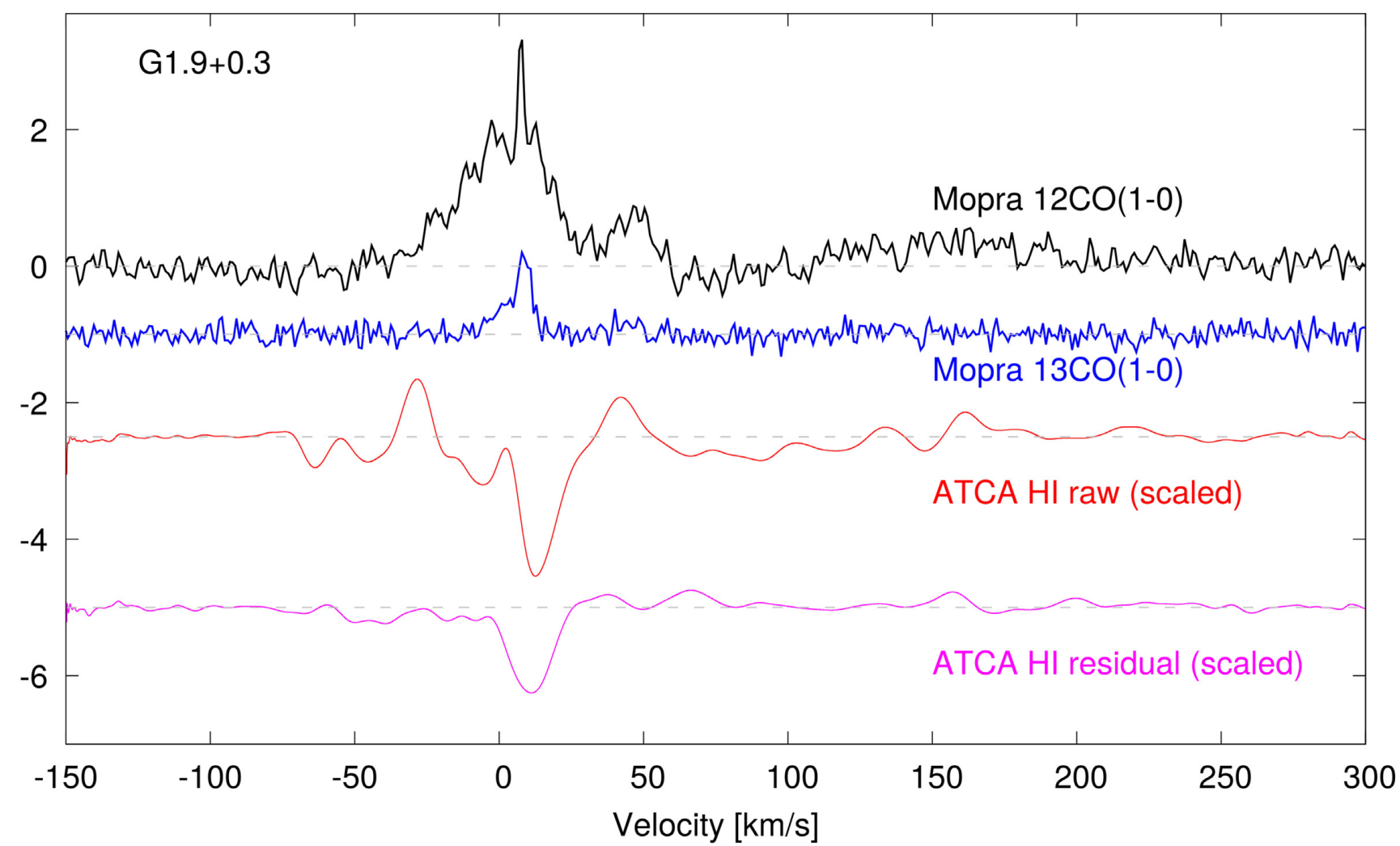

Figure 3. Mopra ${ }^{12} \mathrm{CO}(1-0),{ }^{13} \mathrm{CO}(1-0)$ (Blackwell et al. In Prep.), and ATCA H I spectra from the position of G1.9+0.3. Both a raw H I spectrum (with emission and absorption components) and residual $\mathrm{H}$ I spectrum (i.e. targeting absorption components in the G1.9+0.3 radio continuum only) are shown. H I data were smoothed using a Bézier method.

age. The former is shown in Figs 5 and 6. The areas on Fig. 6 marked with a horizontal green, purple, black, and orange line and labelled 'W', 'N-NE', 'E-SE', and 'S' correspond to the areas introduced by Borkowski et al. (2017), demonstrated in Fig. 7. This allows us to directly compare our radio continuum expansion study to the $\mathrm{X}$-ray estimates. We have included in Table 5 the mean expansion rates and age, as well as the maximum expansion rates and age based thereupon. As expected, these are the regions with the largest expansion rate at both wavebands.

Overall, we have found that the SNR G1.9+0.3 has expanded between 1984 and 2017 at an average rate of $(0.78 \pm 0.09)$ percent $\mathrm{yr}^{-1}$ or $(\sim 8900 \pm 1200) \mathrm{km} \mathrm{s}^{-1}$ which implies a free-expansion age of $(142 \pm 19) \mathrm{yr}$, dating this SNR explosion to mid-to-late 19 th century. This result agrees with previous 


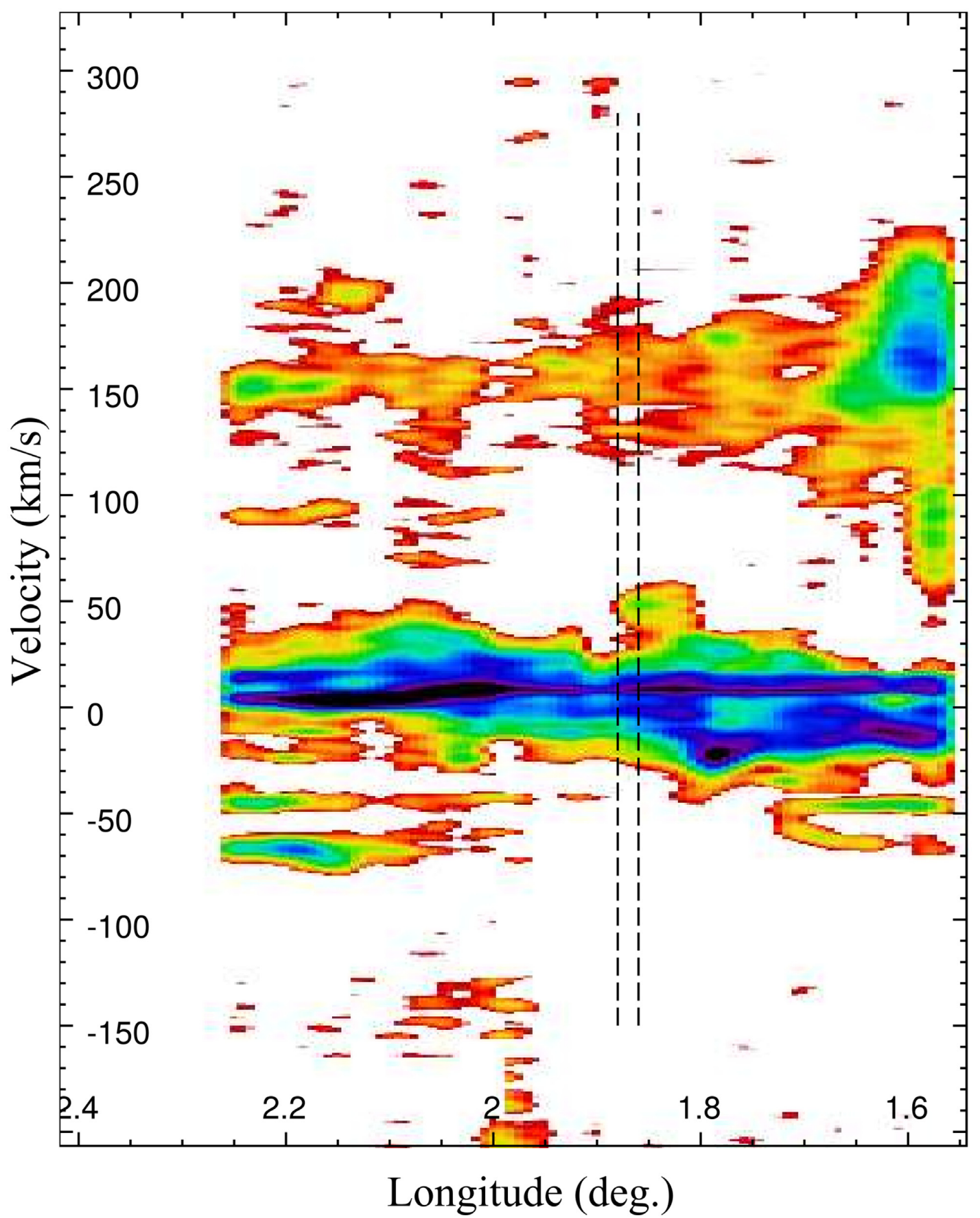

Figure 4. Longitude-Velocity plot of ${ }^{12} \mathrm{CO}(1-0)$ emission. Data are integrated between Galactic latitudes $0.31^{\circ}-0.34^{\circ}$, corresponding to $\mathrm{G} 1.9+0.3$. $\mathrm{CO}$ data are a preliminary result from the Mopra CMZ CO Survey (Blackwell et al. In Prep.). Vertical dashed lines indicate the longitudinal extent of G1.9+0.3.

studies estimating an expansion rate of $(0.642 \pm 0.049)$ per cent $\mathrm{yr}^{-1}$ by Borkowski et al. (2017) and Carlton et al. (2011) and is slightly faster than the $(0.563 \pm 0.078)$ per cent $\mathrm{yr}^{-1}$ measured by De Horta et al. (2014).

\subsection{Polarization}

The polarization of an SNR can be an additional clue towards its age, with young SNR's typically exhibiting a radially orientated magnetic field (Reynolds, Gaensler \& Bocchino 2012). At the same time, polarization is an indicator of the emission mechanism of the SNR, with the presence of polarization indicative of nonthermal emission from high-energy electrons. As a shock evolves and sweeps-up an increasing mass, the shock is expected to decelerate, giving rise to Rayleigh-Taylor instabilities (Gull 1975; Chevalier 1976). As this occurs, the magnetic field lines become increasingly disordered and toroidal, which is apparent from the disordered polarization vectors (Gull 1973; Johnston, McClureGriffiths \& Koribalski 2004). This is largely borne out by similarly young Type Ia SNR's (Milne 1987), as well as those in the Large Magellanic Cloud (LMC, Bozzetto et al. 2014). 


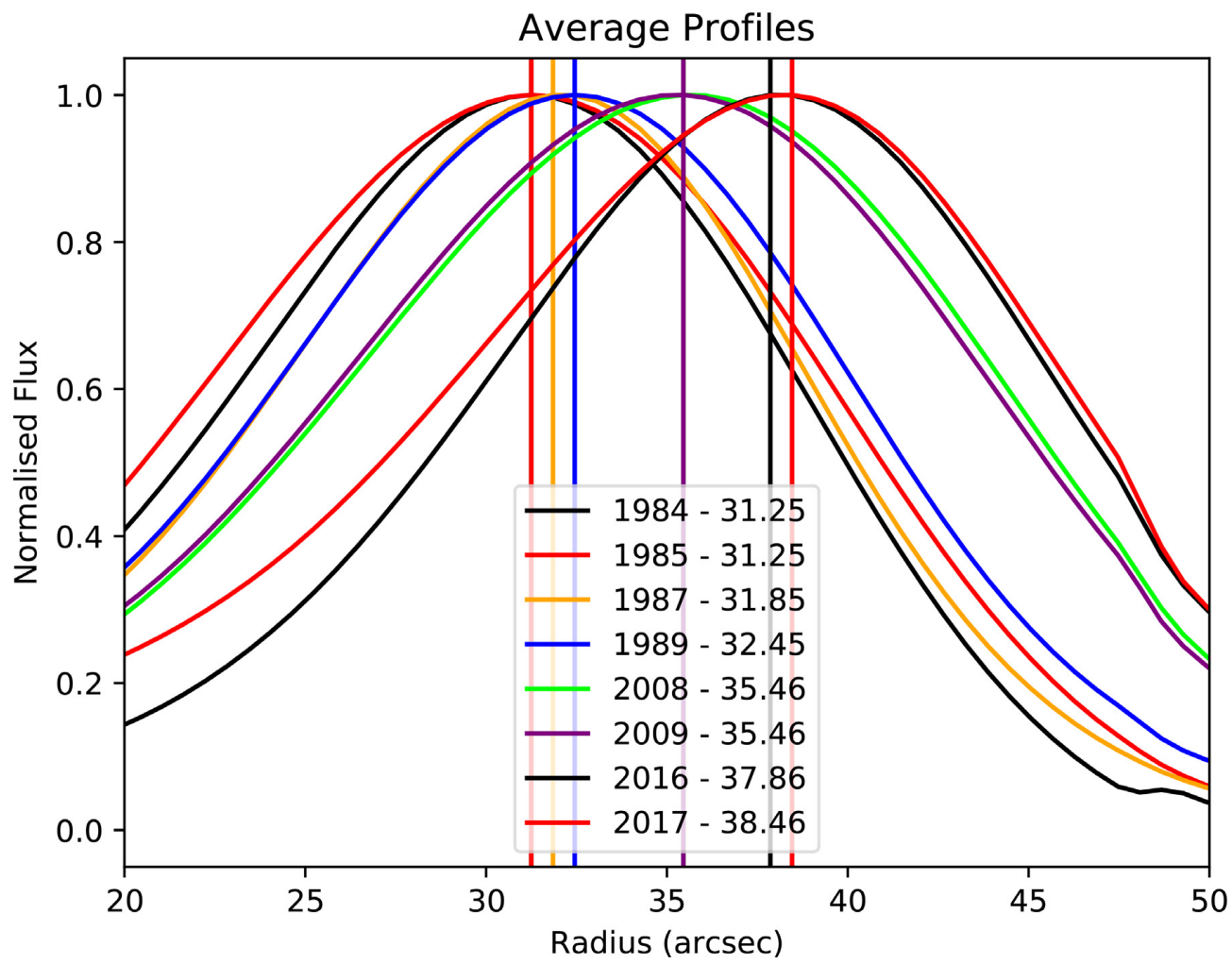

Figure 5. Average shell profiles taken from images synthesized from observations taken from the VLA in 1984, 1985, 1987, 1989, and 2008, and from the ATCA in 2009, 2016, and 2017 (all synthesized to a beam size of $11.12 \operatorname{arcsec} \times 5.32 \mathrm{arcsec}$ ). Line profiles generally show the expected expansion, except in 2016-2017 where the lack of short spacings would seem to be producing a larger average shell profile than expected.

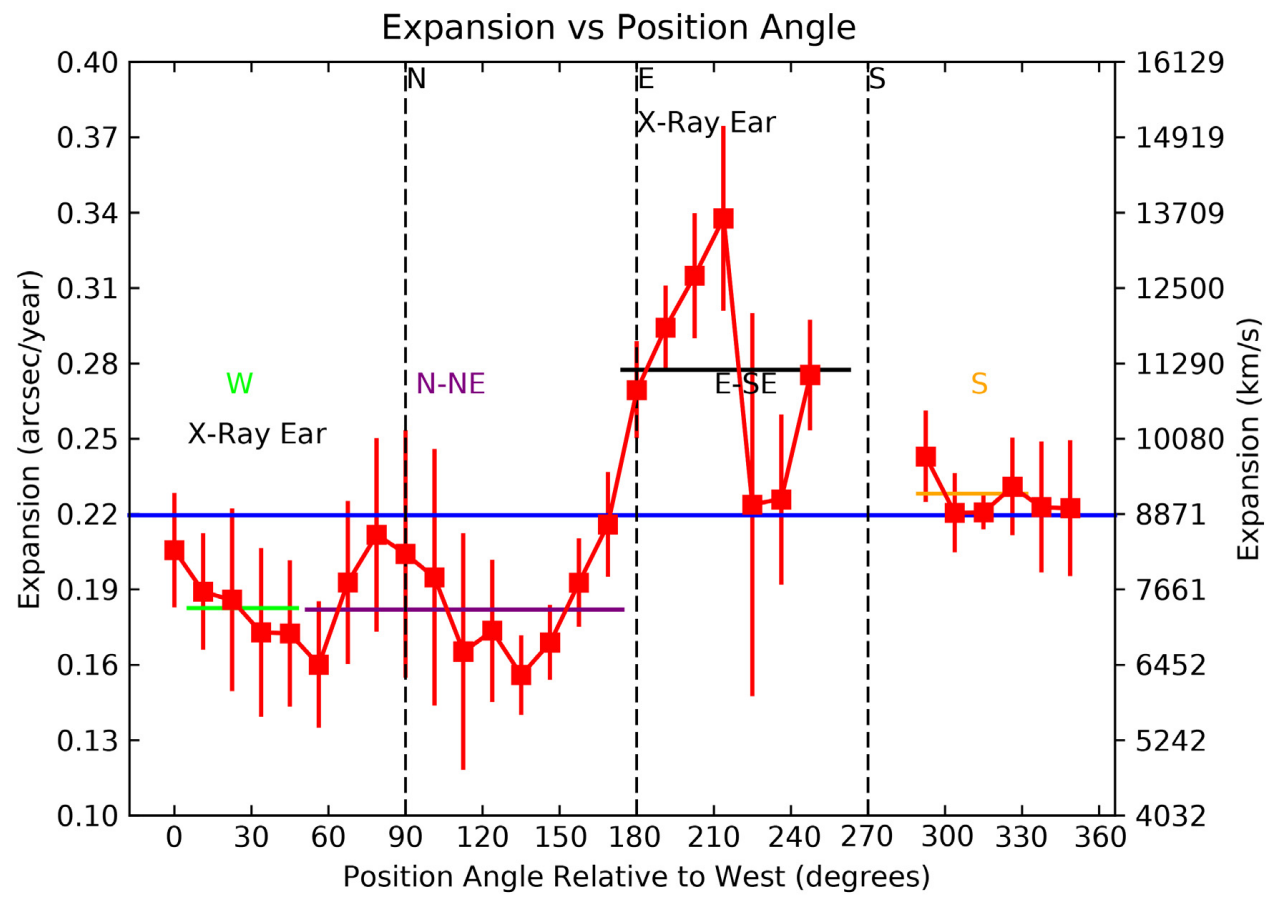

Figure 6. Expansion rates measured using multiple images. We use all radio images accessible from 1984, 1985, 1987, 1989, and 2008 from the VLA and 2009,2016 , and 2017 from the ATCA (all synthesized to a beam size of $11.12 \operatorname{arcsec} \times 5.32 \operatorname{arcsec}$ ). The plot shows the position angle relative to west of which the expansion was measured along the $\mathrm{X}$-axis, and the expansion measured along the $Y$-axis (measured in arcseconds per year on the left and kilometres per second on the right). 


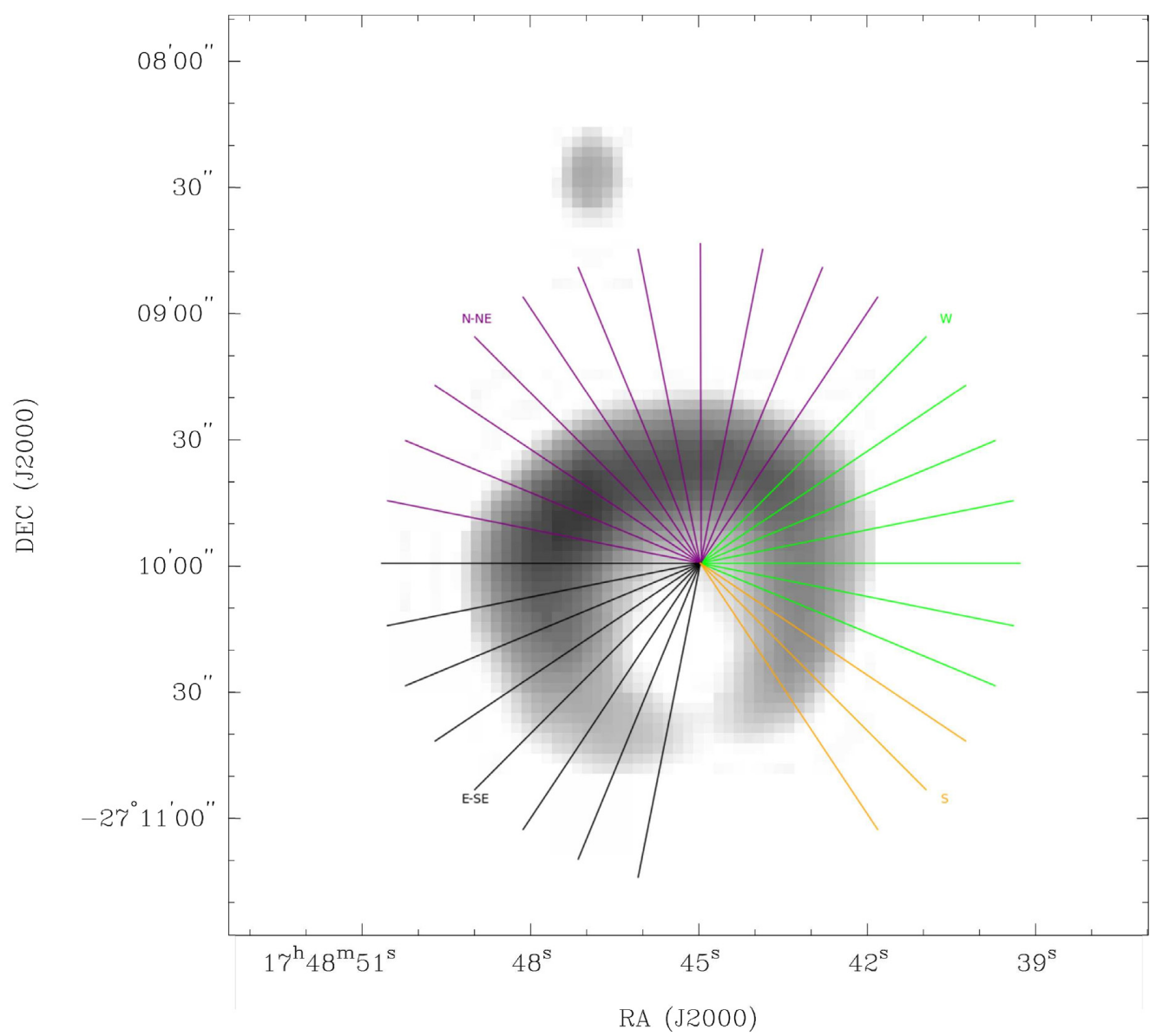

Figure 7. Visualization of the vectors used to calculate the expansion rates in Fig. 6 . The grey scale is the average of all epochs used. The coloured vectors correspond to the west/north-north-east/east-south east/south regions marked in Fig. 6. Vectors extending towards the southern breakout region were discarded.

The ATCA, by default, records the Stokes $Q$ and $U$ parameters required to calculate the polarization vectors. This ensures that as long as the secondary calibrator is observed regularly, and the data are correctly calibrated, then the resultant polarization map will be reliable.

The full resolution polarization images and the RM map have very little signal-to-noise ratio. For a better analysis, we therefore convolved the $Q$ and $U$ maps in the four bands to a common resolution of 9 arcsec. We re-calculated maps of polarized intensity (PI) and polarization angle and the resulting RM at the resolution of 9 arcsec. The resulting RM map and an integrated PI map across the whole band around $5 \mathrm{GHz}$ are displayed in Fig. 8 (left). We also corrected the observed polarization angles for Faraday rotation and added the corrected B-vectors to the PI map in Fig. 8 (right)

In the RM map in Fig. 8 (left), we can see that the eastern (left) shell is dominated by high positive RMs of about +400 to $+600 \mathrm{rad} \mathrm{m}^{-2}$, while the western (right) shell shows mostly low positive RMs of about $100-200 \mathrm{rad} \mathrm{m}^{-2}$. To quantify the distributions of RMs on both of the shells, we plot RM values as a function of right ascension (RA) in Fig. 9. Dashed lines represent the mean values of the eastern and western shells, 411 and $123 \mathrm{rad} \mathrm{m}^{-2}$, respectively. The two concentrations of RMs at about $44^{s}$ and just below $46^{s}$ belong to the weakly polarized northern part of the shell (see left-hand panel in Fig. 8). In Fig. 8 (right), the derived magnetic field vectors in the southern parts of the shells seem to be mostly parallel to the shock normal (parallel from now on), with an average angle of about $20^{\circ}$ to the RA axis. To the north, the eastern shell shows a departure from the parallel magnetic field at a Declination of about $-27^{\circ} 10^{\prime}$ exhibiting close to tangential field (perpendicular to the shock normal; perpendicular from now on). This change in the field structure also coincides with a region of elevated RM value. The parallel magnetic field seems to continue further north for the Western shell, but then changes towards the weakly polarized blobs in the northern part of the shell. This difference in intrinsic magnetic field directions projected to the plane of the sky might be caused by possible interaction of the SNR shell with molecular material in the north.

\subsection{Spectral index}

We estimated the spectral index in Fig. 10 by fitting a line to the 20 flux densities, integrated over the source, between 76.155 and $227.195 \mathrm{MHz}$ measured by the GLEAM project using the Murchison Widefield Array (MWA; Hurley-Walker et al. 2017), an $843 \mathrm{MHz}$ flux density taken from Murphy et al. (2008) measured with the MOST and scaled by 12 per cent to account for the brightening found within their paper, and the flux densities measured from our 2016 ATCA observations. Our flux densities were measured from the 2016 ATCA 2.1, 5, and $9 \mathrm{GHz}$ images, where they had all been convolved to the same beam and pixel size. The $2.1 \mathrm{GHz}$ image was then masked to $20 \sigma\left(\sigma=0.16 \mathrm{mJy}^{\text {beam }}{ }^{-1}\right)$ and then 


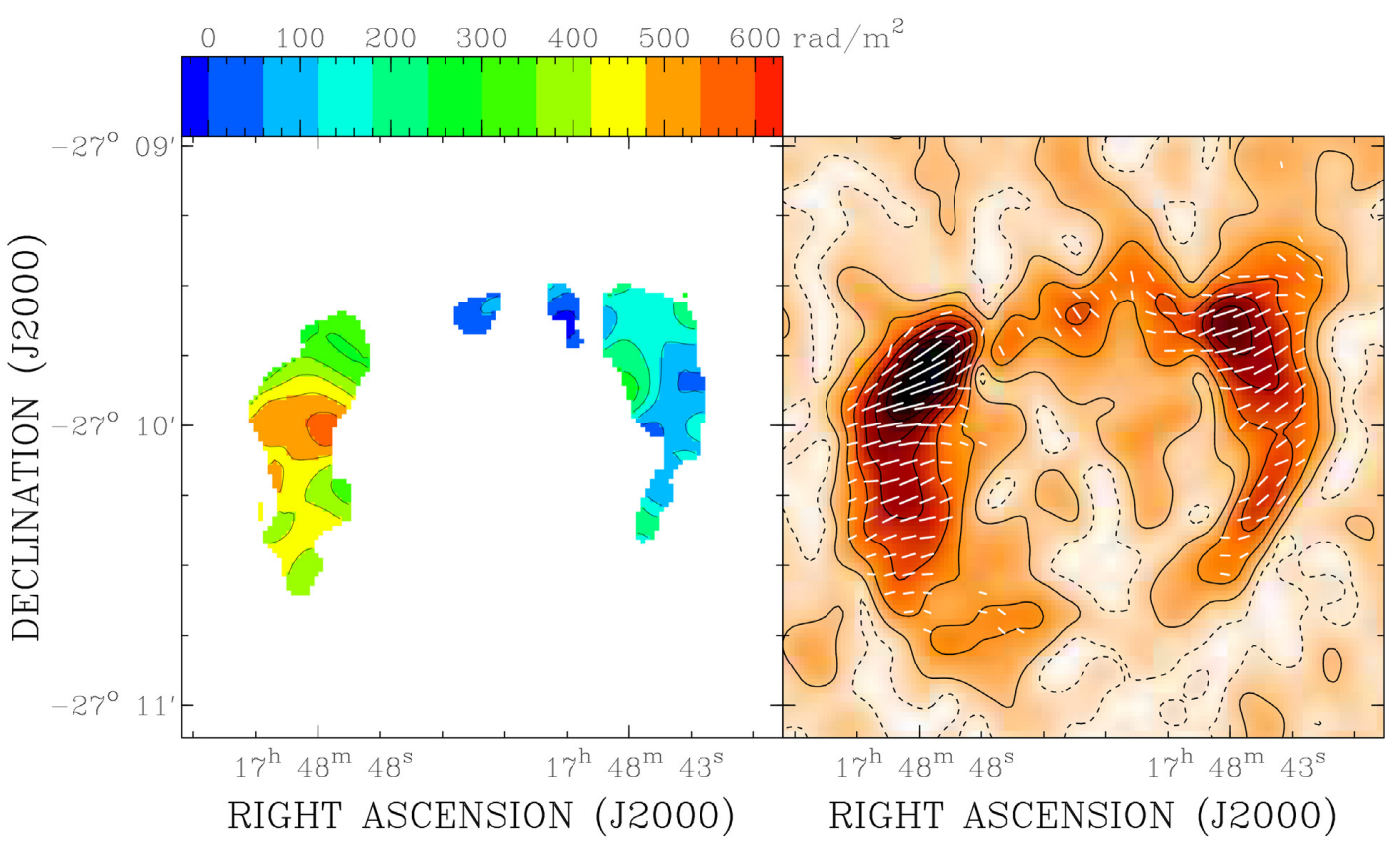

Figure 8. Left: RM map calculated for the SNR G1.9+0.3 after convolving the polarization data to a common resolution of 9 arcsec. Displayed are only RMs calculated for a signal of more than $5 \sigma$ in each band and for RMs with statistical errors of less than $20 \mathrm{rad} \mathrm{m}^{-2}$. Right: PI map at $5 \mathrm{GHz}$ integrated over the whole band. The vectors indicate the magnetic field at the point of origin projected to the plane of the sky. The vectors have been corrected for Faraday rotation.

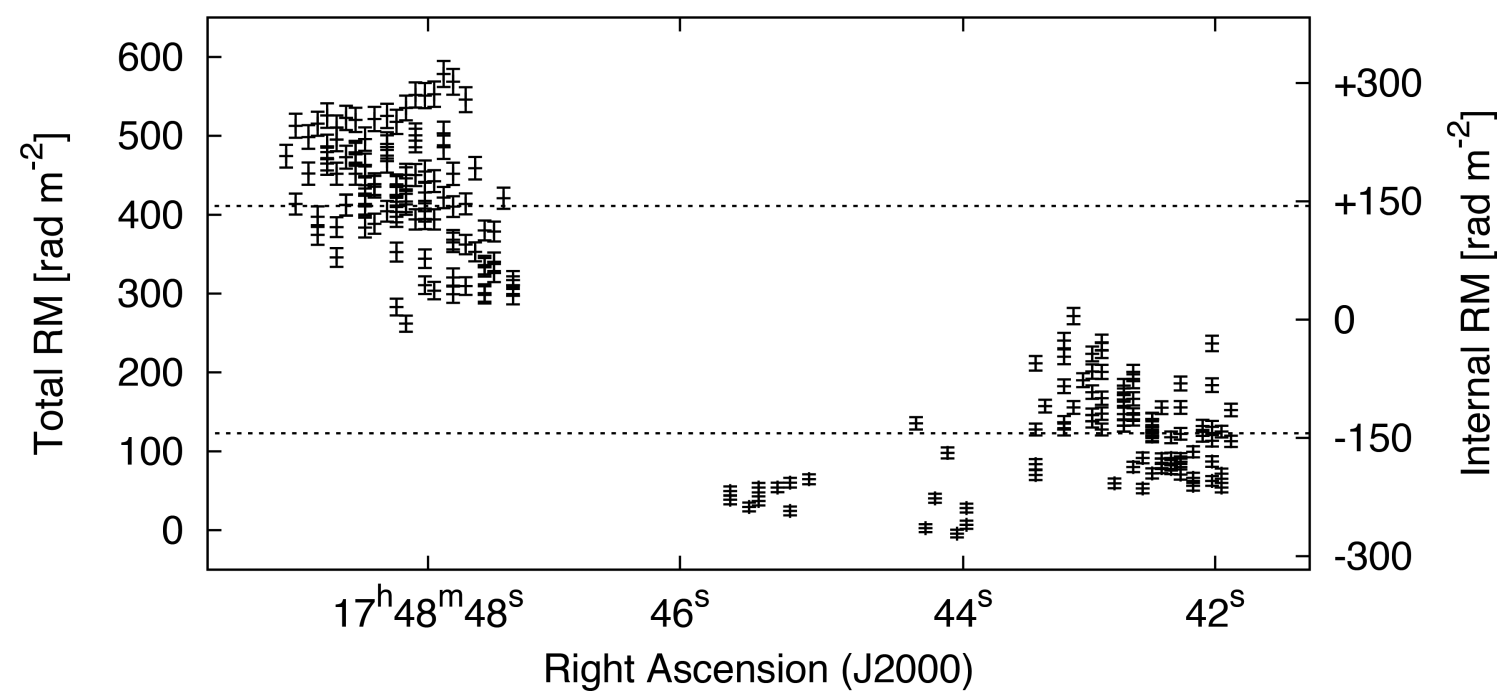

Figure 9. Plot of RMs observed for the SNR G1.9+0.3 as a function of RA. The dashed lines indicate the average RM of $411 \mathrm{rad} \mathrm{m}^{-2}$ for the eastern shell and $123 \mathrm{rad} \mathrm{m}^{-2}$ for the western shell. The two concentrations of RMs at about $44^{s}$ and just below $46^{s}$ belong to the northern shell, presumably interacting with molecular material and were taken into account. The right $Y$-axis labels indicate the RMs after correcting for the assumed Galactic foreground RM of $267 \mathrm{rad} \mathrm{m}^{-2}$.

Table 3. Image details for the three images used in the H I study.

\begin{tabular}{lcccc}
\hline $\begin{array}{l}\text { Image } \\
\text { type }\end{array}$ & $\begin{array}{c}\text { Rms } \\
\left(\mathrm{mJy} \mathrm{beam}^{-1}\right)\end{array}$ & $\begin{array}{c}5 \sigma \\
\left(\mathrm{mJy} \mathrm{beam}^{-1}\right)\end{array}$ & $\begin{array}{c}\text { Resolution } \\
\left(\mathrm{km} \mathrm{s}^{-1}\right)\end{array}$ & $\begin{array}{c}\text { Synthesized } \\
\text { beam and P.A. }\end{array}$ \\
\hline Continuum map & 2.5 & 12.4 & N/A & $\begin{array}{c}14.75 \operatorname{arcsec} \times \\
5.86 \operatorname{arcsec}, 19.4^{\circ}\end{array}$ \\
Total intensity cube & 31.5 & 157.8 & 0.1 & $\begin{array}{c}14.75 \operatorname{arcsec} \times \\
\text { Spectrum cube }\end{array}$ \\
& 3.5 & 17.5 & 1 & $\begin{array}{c}5.86 \operatorname{arcsec}, 19.4^{\circ} \\
5.86 \operatorname{arcsec}, 19.4^{\circ}\end{array}$ \\
\hline
\end{tabular}


Table 4. Epochs analysed for use in expansion study of G1.9+0.3.

\begin{tabular}{|c|c|c|c|c|c|c|c|}
\hline $\begin{array}{l}\text { Observing } \\
\text { date }\end{array}$ & $\begin{array}{l}\text { Project } \\
\text { code }\end{array}$ & Telescope & $\begin{array}{c}\text { Array } \\
\text { configuration }\end{array}$ & $\begin{array}{l}\text { Bandwidth } \\
(\mathrm{MHz})\end{array}$ & $\begin{array}{c}\text { Frequency } \\
v(\mathrm{GHz})\end{array}$ & $\begin{array}{l}\text { Original synthesized } \\
\text { beam }\end{array}$ & $\begin{array}{c}\text { Original position } \\
\text { angle }\end{array}$ \\
\hline $26 / 05 / 1984$ & AG0146 & VLA & $\mathrm{C}$ & 50 & 4.8351 and 4.8851 & $7.76 \operatorname{arcsec} \times 3.43 \operatorname{arcsec}$ & $-6.2^{\circ}$ \\
\hline $16 / 04 / 1985$ & AG0184 & VLA & $\mathrm{B}$ & 50 & 1.4649 and 1.5149 & $2.78 \operatorname{arcsec} \times 1.11 \operatorname{arcsec}$ & $-5.5^{\circ}$ \\
\hline 22/02/1987 & AB0407 & VLA & $\mathrm{CD}$ & 50 & 4.8351 and 4.8851 & $10.05 \operatorname{arcsec} \times 9.27 \operatorname{arcsec}$ & $+64.1^{\circ}$ \\
\hline 23/06/1989 & AB0544 & VLA & $\mathrm{BC}$ & 50 & 4.8351 and 4.8851 & $8.03 \operatorname{arcsec} \times 3.35 \operatorname{arcsec}$ & $-26.3^{\circ}$ \\
\hline $12 / 03 / 2008$ & AG0793 & VLA & $\mathrm{C}$ & 50 & 4.8351 and 4.8851 & $2.78 \operatorname{arcsec} \times 1.11 \operatorname{arcsec}$ & $-5.5^{\circ}$ \\
\hline 20/01/2009 & C1952 & ATCA & EW $352+6 C$ & 128 & 4.5440 and 5.1840 & $11.12 \operatorname{arcsec} \times 5.32 \operatorname{arcsec}$ & $-0.8^{\circ}$ \\
\hline $19 / 02 / 2016$ & C1952 & ATCA & EW $352+6 B$ & 4096 & 2.1000 and 5.0000 & $7.74 \operatorname{arcsec} \times 3.51 \operatorname{arcsec}$ & $-5.5^{\circ}$ \\
\hline $20 / 05 / 2017$ & C1952 & ATCA & $6 \mathrm{~A}$ & 4096 & 2.1000 and 5.0000 & $1.80 \operatorname{arcsec} \times 0.68 \operatorname{arcsec}$ & $+2.4^{\circ}$ \\
\hline
\end{tabular}

Table 5. Expansion study results - matched resolution (11.12 arcsec $\times 5.32$ arcsec ) using images synthesized from observations from 1984,1985 , 1987,1989 , 2008, 2009, 2016, and 2017. The regions are as defined in Fig. 6.

\begin{tabular}{|c|c|c|c|c|c|c|c|c|}
\hline Region & $\begin{array}{c}\text { Average } \\
\text { expansion } \\
\left(\operatorname{arcsec} \mathrm{yr}^{-1}\right)\end{array}$ & $\begin{array}{c}\text { Maximum } \\
\text { expansion } \\
\left(\operatorname{arcsec} \mathrm{yr}^{-1}\right)\end{array}$ & $\begin{array}{c}\text { Average } \\
\text { expansion } \\
\left(\text { per ecnt } \mathrm{yr}^{-1}\right)\end{array}$ & $\begin{array}{c}\text { Maximum } \\
\text { expansion } \\
\left.\text { (per cent } \mathrm{yr}^{-1}\right)\end{array}$ & $\begin{array}{l}\text { Average } \\
\text { expansion } \\
\left(\mathrm{km} \mathrm{s}^{-1}\right)\end{array}$ & $\begin{array}{c}\text { Maximum } \\
\text { expansion } \\
\left(\mathrm{km} \mathrm{s}^{-1}\right)\end{array}$ & $\begin{array}{c}\text { Average } \\
\text { age } \\
\text { (yr) }\end{array}$ & $\begin{array}{c}\text { Minimum } \\
\text { age } \\
(\mathrm{yr})\end{array}$ \\
\hline Overall & $0.22 \pm 0.03$ & $0.34 \pm 0.08$ & $0.78 \pm 0.09$ & $1.20 \pm 0.23$ & $8854 \pm 1195$ & $13616 \pm 3075$ & $142 \pm 19$ & $93 \pm 7$ \\
\hline West & $0.18 \pm 0.03$ & $0.19 \pm 0.04$ & $0.65 \pm 0.09$ & $0.67 \pm 0.11$ & $7364 \pm 1248$ & $7627 \pm 1462$ & $155 \pm 18$ & $149 \pm 15$ \\
\hline North & $0.18 \pm 0.03$ & $0.21 \pm 0.05$ & $0.56 \pm 0.10$ & $0.65 \pm 0.16$ & $7338 \pm 1290$ & $8539 \pm 2059$ & $178 \pm 18$ & $153 \pm 11$ \\
\hline East & $0.28 \pm 0.03$ & $0.34 \pm 0.08$ & $0.84 \pm 0.10$ & $1.02 \pm 0.23$ & $11181 \pm 1324$ & $13616 \pm 3075$ & $119 \pm 17$ & $98 \pm 7$ \\
\hline South & $0.23 \pm 0.01$ & $0.24 \pm 0.02$ & $0.90 \pm 0.04$ & $0.96 \pm 0.06$ & $9196 \pm 545$ & $9798 \pm 731$ & $111 \pm 41$ & $104 \pm 31$ \\
\hline
\end{tabular}

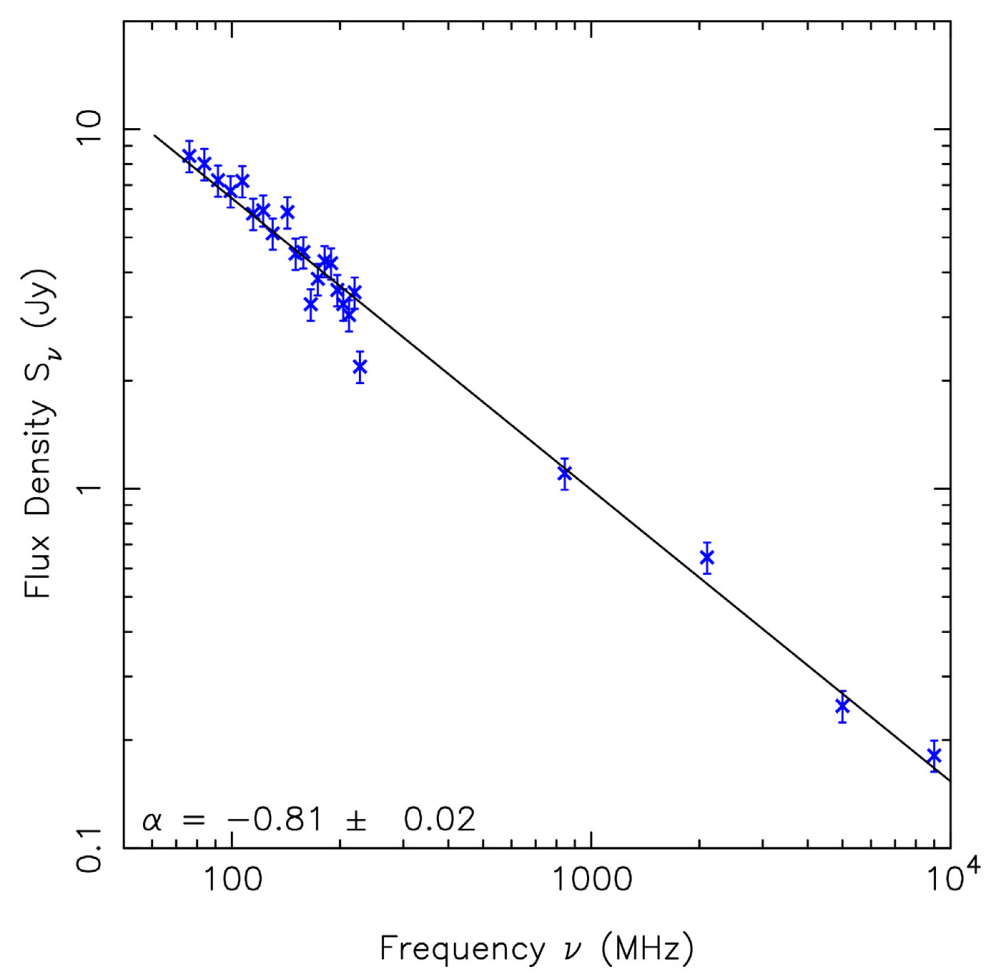

Figure 10. Integrated spectral energy distribution of SNR G1.9+0.3 containing 20 flux densities from the GLEAM survey (Hurley-Walker et al. 2019; see Table 6 for the individual flux density measurements used) from 76 to $227 \mathrm{MHz}$, one $843 \mathrm{MHz}$ flux density measured using the MOST (with the flux density scaled based on the radio brightening found by Murphy et al. 2008) and the 2.1, 5.0, and 9.0 GHz flux densities measured using the 2016 ATCA observations.

used to mask the 5 and $9 \mathrm{GHz}$ images to ensure the flux density measurements were taking into account the same pixels for all images. All flux densities used in Fig. 10 are listed in Table 6.

Using these flux densities, we obtain a spectral index of $-0.81 \pm 0.02-$ shown in Fig. 10 - which is comparable to the spectral index of $-0.93 \pm 0.23$ calculated by LaRosa et al. (2000).
However, it is steeper than the spectral index calculated by Green et al. (2008) of $-0.62 \pm 0.06$. Green et al. (2008) note that their estimated spectral index differs from values in the literature, and instead suggest a spectral index of $\approx-0.7$, which is closer to the spectral index estimated here. With a spectral index of $-0.81 \pm 0.02$, G1.9+0.3 has one of the steepest spectral indexes known in our 
Table 6. Flux densities, integrated over G1.9+0.3 measured using the MWA, MOST, and ATCA. All flux density measurements are assumed to have an error of 10 percent, with the MOST $843 \mathrm{MHz}$ flux density scaled up by 12 per cent to account for the brightening found by Murphy et al. (2008).

\begin{tabular}{lrr}
\hline Instrument & $v(\mathrm{MHz})$ & $S(\mathrm{Jy})$ \\
\hline MWA & 76.155 & 8.438 \\
MWA & 83.835 & 8.022 \\
MWA & 91.515 & 7.216 \\
MWA & 99.195 & 6.734 \\
MWA & 106.875 & 7.184 \\
MWA & 114.555 & 5.827 \\
MWA & 122.235 & 5.953 \\
MWA & 129.915 & 5.135 \\
MWA & 142.715 & 5.885 \\
MWA & 150.395 & 4.512 \\
MWA & 158.075 & 4.556 \\
MWA & 165.755 & 3.261 \\
MWA & 173.435 & 3.838 \\
MWA & 181.115 & 4.301 \\
MWA & 188.795 & 4.240 \\
MWA & 196.475 & 3.575 \\
MWA & 204.155 & 3.263 \\
MWA & 211.835 & 3.044 \\
MWA & 219.515 & 3.518 \\
MWA & 227.195 & 2.188 \\
MOST & 843.000 & 1.104 \\
ATCA & 2100.000 & 0.645 \\
ATCA & 5000.000 & 0.249 \\
ATCA & 9000.000 & 0.181 \\
\hline & & \\
\hline
\end{tabular}

Galaxy and Small Magellanic Clouds and LMCs (Bozzetto et al. 2017; Maggi et al. 2019).

Such a steep spectral index is characteristic of young SNRs (Urošević 2014). The steep spectra of young SNRs can be caused by quasi-perpendicular magnetic field geometry (Bell, Schure \& Reville 2011), turbulent magnetic field amplification (Bell, Matthews \& Blundell 2019), Alfvénic drift effect (Jiang, Zhang \& Fang 2013), and by pure NLDSA effects which efficiently produce strong magnetic field amplification (Pavlović 2017).

By using the radio spectrum from the ATCA, MOST, and MWA in conjunction with the distance of $\sim 8.5 \mathrm{kpc}$ and a diameter of $95 \mathrm{arcsec}$, we can calculate the total flux density at $1 \mathrm{GHz}(0.99 \mathrm{Jy})$ surface Brightness $\left(0.60 \times 10^{-19} \mathrm{~W}\left(\mathrm{~m}^{2} \mathrm{~Hz} \mathrm{SR}\right)^{-1}\right)$ and luminosity between $10 \mathrm{MHz}$ and $100 \mathrm{GHz}\left(0.89 \times 10^{26} \mathrm{~W} \mathrm{~Hz}^{-1}\right)$.

The spectral index map presented in Fig. 11 is the product of the MIRIAD task MFSPIN, with our 2.1, 5.0, and 9.0 GHz data combined into a single $u v$ file before imaging, with the $u v$ range tapered to ensure matching coverage across all bands. This image was then masked to $20 \sigma$ - where $\sigma$ is measured to be $0.05 \mathrm{mJy}_{\text {beam }}^{-1}$, before the MIRIAD task MFSPIN was run, allowing for the final $20 \sigma$ spectral index map in Fig. 11 to be created. The steepest section of this spectral index map is measured at -1.07 in the northern region, where we also see somewhat randomized polarization vectors (Fig. 8) and extreme radio brightening in Section 3.5. The average spectral index measured across the SNR is -0.61 , with a standard deviation of 0.18 .

We would not necessarily expect the spectral index presented in Fig. 10 to be equivalent to the average spectral index measured from the spectral index map presented in Fig. 11, as the spectral index presented in Fig. 10 is effectively taking the average across the map unweighted by flux density, whereas the spectral index map in Fig. 11 is effectively weighted by the flux density.

\subsection{Radio brightening}

In Fig. 12, a striking difference in the northern shock position was observed, with the 2017 G1.9+0.3 image extending farther north than 2016 image. By taking our $2.1 \mathrm{GHz}$ observations from 2016 March with the ATCA in a $6 \mathrm{~B}$ configuration and our $2.1 \mathrm{GHz}$ observations from 2017 May with the ATCA in a 6A configuration imaged to the same beam size, we can construct an image comparing the flux density levels from each. Fig. 13 is an image that shows the relative radio flux density brightening, calculated using equation (1) across the 14 month period.

Brightening $=\frac{S_{2017}-S_{2016}}{S_{2016}} \times 100$

We mask the resulting image so that any pixel is masked if it is below $10 \sigma$ in either the 2016 image $\left(\sigma=0.16 \mathrm{mJy}^{\text {beam }}{ }^{-1}\right.$, or in the 2017 image $\left(\sigma=0.28 \mathrm{mJy}^{\text {beam }}{ }^{-1}\right)$.

The brightness percentage map in Fig. 13 has an average increase of 1.95 percent. There is a minimum of -57.08 percent, and a maximum brightening of 228.30 per cent along the northern region where we found decreased expansion in Section 3.2, a less-ordered polarization field as can be seen in Fig. 8 (right) and a steep spectral index in Section 3.4.

By interpolating this 1.95 percent brightening rate from the measured 14-12 months, we find an average brightening of $(1.67 \pm 0.35)$ per cent $\mathrm{yr}^{-1}$ (consistent with the value of 1.8 per cent predicted by simulations in Pavlović 2017), minimum brightening rate of $\sim-50$ percent $\mathrm{yr}^{-1}$, and a maximum brightening rate of $\sim 200$ percent $\mathrm{yr}^{-1}$. Our new average result is in line with the $\left(1.22_{-0.16}^{+0.24}\right)$ percent $\mathrm{yr}^{-1}$ measured by Murphy et al. (2008) with their study over $20 \mathrm{yr}$ using the MOST, and the $\approx 2$ per cent increase per year measured by Green et al. (2008) with the VLA.

\section{DISCUSSION}

SNR G1.9+0.3 is unique, in that it is the youngest known Galactic Type Ia SNR. As such, it provides a window into the evolution of the young SNRs. While some of the observations demonstrated are within the expected and currently measured bounds - radio expansion rate and spectral index - some observations differ.

The RM map shown in Fig. 9 is indicative of one of the divergences from current observations. A similar behaviour for the distribution of RMs on the two opposite shells of an SNR were found for the SNR G296.5+10.0 by Harvey-Smith et al. (2010). They found relatively constant RMs of opposite sign on both shells of the SNR. As G1.9+0.3 is estimated to be further away, and closer to the centre of our Galaxy, we would expect quite significant foreground $\mathrm{RM}$. Assuming a positive foreground $\mathrm{RM}$ of about $+267 \mathrm{rad} \mathrm{m}^{-2}$, the internal eastern and western shell RMs would be roughly matched with opposite signs of about $\pm 144 \mathrm{rad} \mathrm{m}^{-2}$ (see Fig. 9). We tried to analyse the foreground RM by comparing RMs of pulsars and extragalactic sources, at short angular distances from the SNR. For the pulsars, we are using the 'The Australia Telescope National Facility Pulsar Catalogue' (Manchester et al. 2005). ${ }^{2}$ We find two pulsars with known $\mathrm{RM}$ of +421 and $+916 \mathrm{rad} \mathrm{m}^{-2}$ within $1^{\circ}$ of G1.9+0.3 (Han et al. 2018) with dispersion measure (DM) distances

\footnotetext{
${ }^{2} \mathrm{http}: / /$ www.atnf.csiro.au/research/pulsar/psrcat/
} 


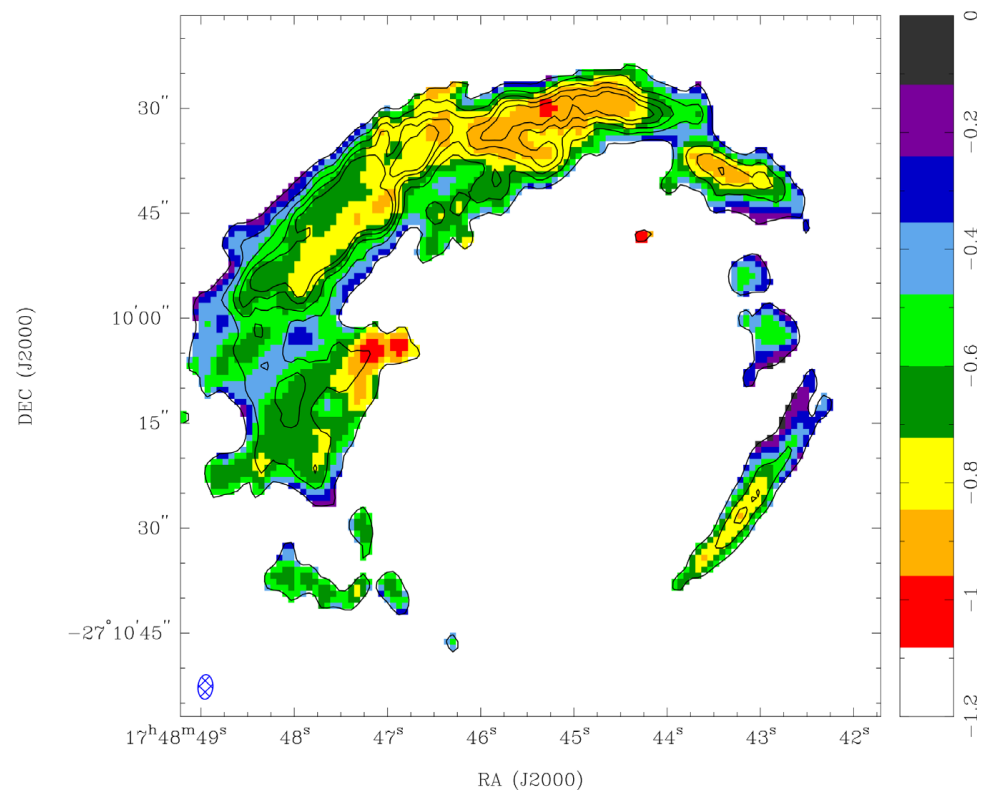

Figure 11. Spectral index map produced from observations from 2016 using the ATCA. This plot shows an average of -0.61 , minimum value of -1.07 , and standard deviation of 0.18 .

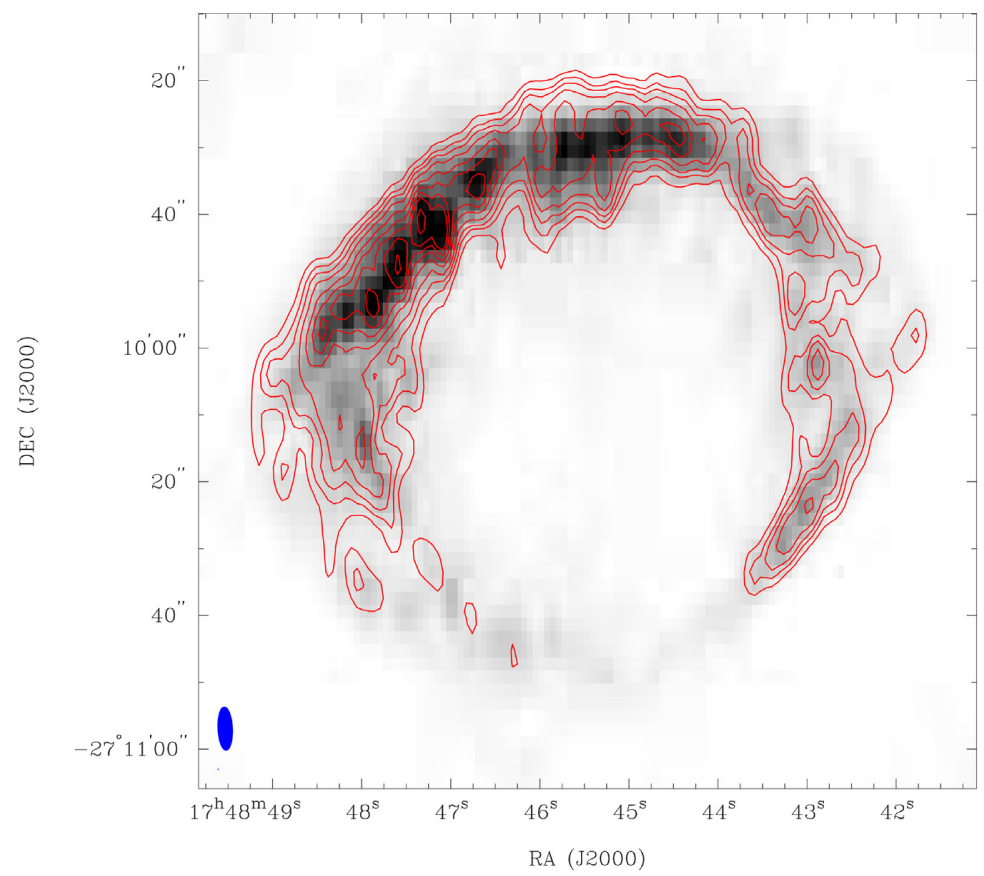

Figure 12. 2016 6B array observations at $2.1 \mathrm{GHz}$ as the grey-scale image, with contours taken from our $20176 \mathrm{~A}$ observations at $2.1 \mathrm{GHz}$. Contour levels are $3 \sigma, 5 \sigma, 7 \sigma, 10 \sigma, 15 \sigma, 20 \sigma, 25 \sigma, 30 \sigma$, and $40 \sigma\left(\sigma=0.16 \mathrm{mJy} \mathrm{Beam}^{-1}\right)$.

of 5.2 and $4.2 \mathrm{kpc}$, respectively (Yao, Manchester \& Wang 2017). There are two linearly polarized background extragalactic sources with RMs of +806 and $+638 \mathrm{rad} \mathrm{m}^{-2}$ within $1^{\circ}$ of $\mathrm{G} 1.9+0.3$ taken from a catalog of Faraday RM of point sources published by $\mathrm{Xu} \&$ Han (2014). ${ }^{3}$ These values indicate that the RMs in this direction are varying a lot and are in general positive with a high amplitude. A foreground $\mathrm{RM}$ of $+267 \mathrm{rad} \mathrm{m}^{-2}$ is certainly not unexpected, but

${ }^{3}$ http://zmtt.bao.ac.cn/RM/searchGRM.html is low compared to other RMs from the same direction. However, it also cannot be excluded that the difference in the RM between the eastern and western shells is simply due to variations of the foreground RM.

Harvey-Smith et al. (2010) showed that such a behaviour of the RM in G296.5+10.0 can be explained by an SNR expanding inside an azimuthal/toroidal magnetic field in the stellar wind of the progenitor star of the SN explosion. In this case, magnetic field lines would be wrapped around the expanding SNR in the equatorial region which in the projected picture will show up as a quasi-radial 


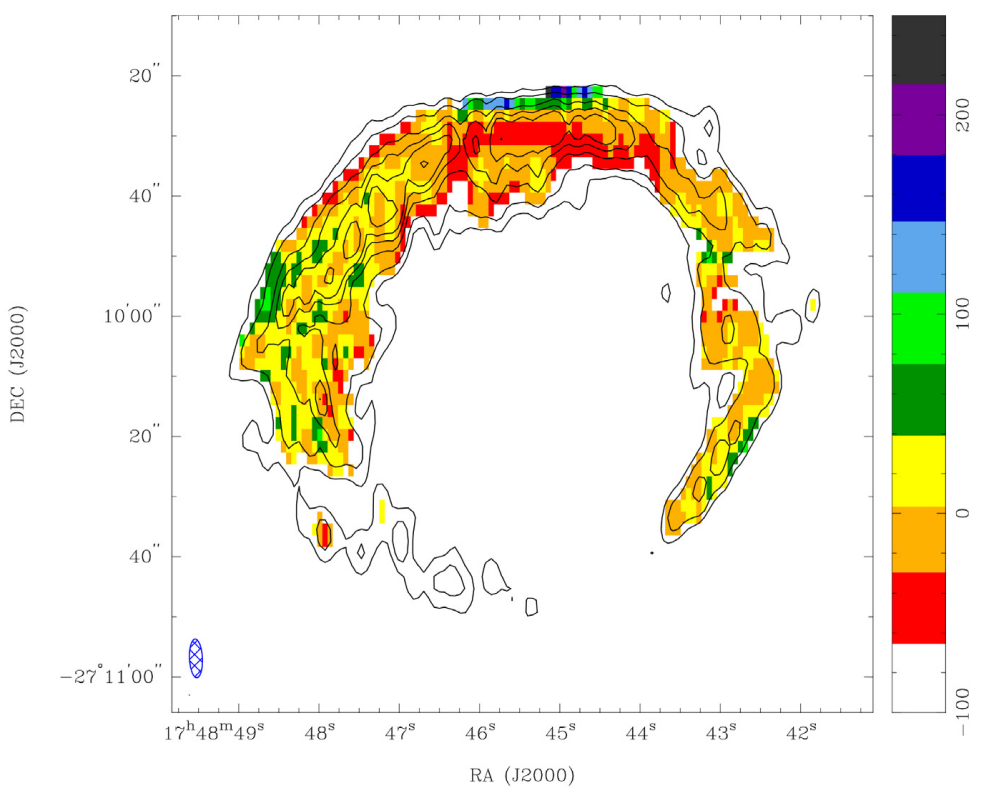

Histogram of Values from the 2016v2017 Brightening Map

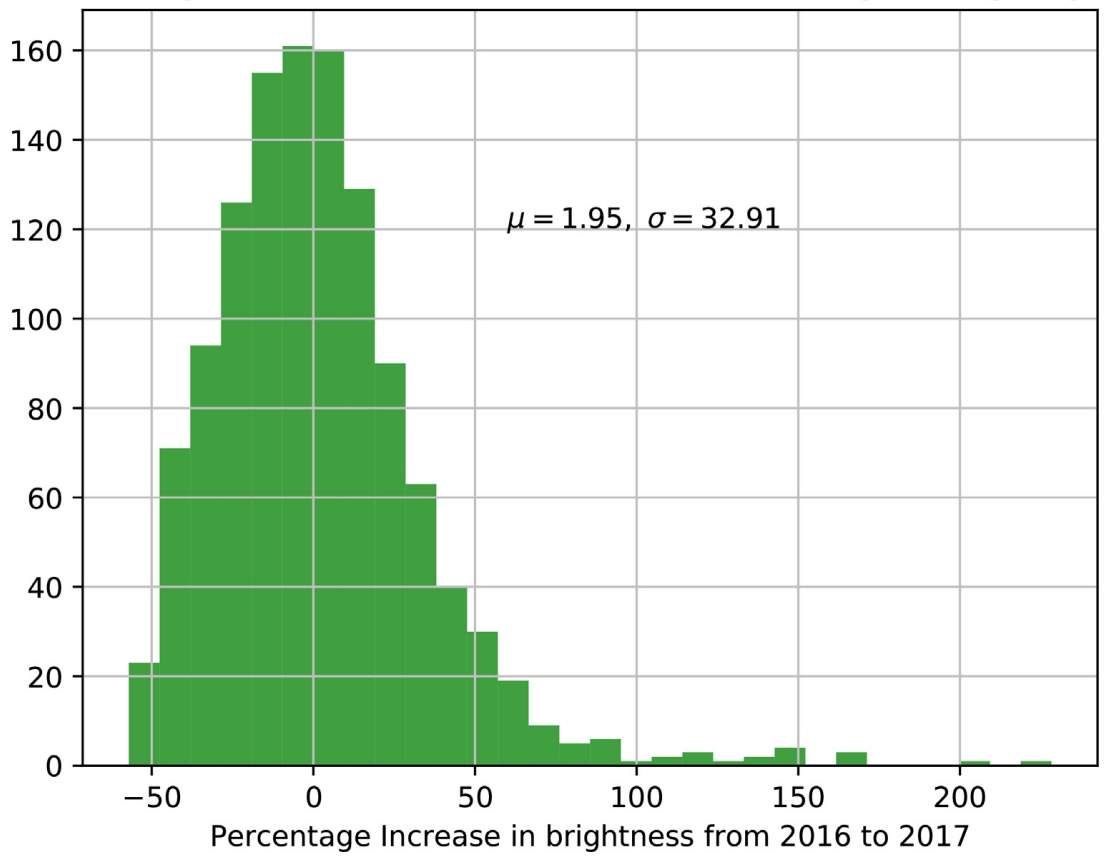

Figure 13. Radio brightening map (top) produced from the 2016 6B array observations and $20176 \mathrm{~A}$ array observations using the ATCA. Both 2016 and 2017 observations were masked below $10 \sigma\left(2016, \sigma=0.16 \mu \mathrm{Jy}_{\text {beam }}^{-1}\right.$ and 2017, $\left.\sigma=0.28 \mu \mathrm{Jy} \mathrm{beam}^{-1}\right)$. Average brightening in radio is found to be 1.95 per cent with a minimum of -57.08 per cent, maximum of 228.3 per cent and a standard deviation of 32.31 . Contours taken from the $20162.1 \mathrm{GHz} 6 \mathrm{~B}$ image at $5 \sigma$, $10 \sigma, 20 \sigma, 30 \sigma, 50 \sigma$, and $80 \sigma\left(\sigma=0.16 \mathrm{mJy}_{\text {beam }}{ }^{-1}\right)$. Histogram (bottom) showing the counts of pixel intensities from the radio brightening map (top). The region of highest flux density in the northern region shows a dimming due to the shift of the peak in the northern region moving further north.

field inside the shell with the decrease of the in-plane component towards the edge of the remnant. This roughly corresponds to what we see in G1.9+0.3 (see Fig. 8, left) if the equatorial plane is tilted by $20^{\circ}$ with respect to the RA axis. From such a magnetic field configuration, we would expect the RMs to have the same amplitude on both shells but opposite sign. Harvey-Smith et al. (2010) simulated the expansion of a late time stellar wind of the progenitor star in order to calculate the RM that would be imposed on background linearly polarized emission. They studied several different progenitor stars and showed that the red super giant (RSG) wind can be responsible for the levels of the RM in G296.5+10.0. Using the same formalism, it is easy to demonstrate that this could also be a plausible scenario to explain the RM distribution in $\mathrm{G} 1.9+0.3$.

However, the RSG wind and toroidal magnetic field hypothesis does not explain all aspects of this SNR.

First, to reach the size of G1.9+0.3 in this scenario, the age of the SNR would need to be closer to $\sim 400$ yr old (Dwarkadas 2005; 
Telezhinsky et al. 2013), because of the expected shock speed of an SNR expanding inside the stellar wind of the progenitor star. A superluminous $\mathrm{SN}$ explosion would be required to reach the expansion velocity and size observed in G1.9+0.3. Additionally, the lower shock speed anticipated from this scenario would result in a lower maximum electron energy, which would be difficult to reconcile with the observed X-ray emission. The RSG scenario should produce more thermal X-ray emission than reported by Borkowski et al. (2013).

Second, the RSG scenario would make G1.9+0.3 a twin of Cassiopeia A, which would raise the question - why are the respective morphologies so different?

Third, a toroidal magnetic field in the stellar wind of the progenitor star would be parallel to the shock, which makes particle acceleration at the shock very inefficient (Völk, Berezhko \& Ksenofontov 2003; Caprioli \& Spitkovsky 2014) which in turn results in a lack of magnetic field amplification by the cosmic rays (CRs). This contradicts the explanation of the synchrotron emission from the SNR. Theoretical modelling requires a high magnetic field downstream of the forward shock with estimates ranging from $180 \mu \mathrm{G}$ (Brose et al. 2019) to >300 $\mu \mathrm{G}$ (Pavlović 2017; Urošević, Pavlović \& Arbutina 2018) which in turn are in agreement with an equipartition magnetic field of 180-273 $\mu \mathrm{G}$ (De Horta et al. 2014).

Finally, simulations presented in Brose et al. (2019) strongly suggest that radio emission predominantly originates from the reverse shock, which means that it is not sensitive to the the structure of the magnetic field upstream of the forward shock. A dedicated study of polarization of the X-ray emission would be extremely important to solve some of these discrepancies.

Unexpected radio brightening was also observed in the northern region. Uchiyama et al. (2007) have previously observed localized brightening (and fading; explained by synchrotron cooling) of X-ray emission in RX J1713.7 - 3946 on a one-year time-scale. This was said to be synchrotron emission from electrons quickly accelerated by the diffusive shock acceleration process, and the high energy of the variable emission indicated magnetic field amplification in that region of the SNR shock, which corresponds to dense molecular clumps (Sano et al. 2010; Maxted et al. 2013; Sano et al. 2015). Therefore, the largest brightening increase in the northern parts of G1.9+0.3 (Fig. 13) may indicate regions of shock interaction with a highly inhomogeneous ISM. This motivates future X-ray studies probing small-scale X-ray features as well as future ISM observations at arcseconds resolution (e.g. using ALMA, e.g. see Sano et al. 2019, or ATCA) to identify shock/ISM interactions.

In addition to exhibiting short-time-scale X-ray brightening linked to particle acceleration, SNR RX J1713.7-3946 is a strong TeV gamma-ray source (Aharonian et al. 2007; H.E.S.S. Collaboration et al. 2018) and has been the subject of a number of investigations that search for signatures of $\mathrm{CR}$ hadrons accelerated within the SNR shell (e.g. Fukui et al. 2012). The localized radio brightening of G1.9+0.3 discovered in our study highlights the potential of $\mathrm{G} 1.9+0.3$ as a powerful particle accelerator as well. Indeed, as argued in Section 3.3, the B-field amplification implied by strong synchrotron X-ray emission can be naturally explained by CRs (e.g. CR streaming instabilities). It follows that this object is a key target in CR origin studies with future high-sensitivity TeV gamma-ray observations of the Cherenkov Telescope Array (see Cherenkov Telescope Array Consortium et al. 2019).

\section{CONCLUSION}

This radio study of the youngest known Galactic SNR has used new ATCA observations in 2016 and 2017 and preliminary observations from the Mopra and MWA telescopes to examine the flux density, distance, spectral index, polarization, brightening, and expansion of the G1.9+0.3 shell. Our main findings are:

(i) Our H I and CO study is consistent with a distance of $8.5 \mathrm{kpc}$;

(ii) G1.9+0.3 has a mean expansion (in radio continuum) rate of $(0.78 \pm 0.09)$ per cent $\mathrm{yr}^{-1}$ over a $31-\mathrm{yr}$ period, equivalent to $(8800 \pm 1200) \mathrm{km} \mathrm{s}^{-1}$ at a distance of $8.5 \mathrm{kpc}$;

(iii) There are very different RM values towards the two socalled (east and west) 'ears' of G1.9+0.3. The expansion into the stellar wind of an RGS star progenitor could potentially explain the polarization characteristics we observe for SNR G1.9+0.3. This hypothesis, however, would have difficulties explaining hydrodynamic properties of the remnant and its observed X-ray emission;

(iv) G1.9+0.3 has a global spectral index of $(-0.81 \pm 0.02)$, which steepens to $\sim-1$ towards the remnant's north, where we also find increased brightening of up to 195 percent, consistent with brightening due to expansion into an inhomogeneous ISM;

(v) There is an average radio brightening of $(1.67 \pm 0.35)$ per cent $\mathrm{yr}^{-1}$.

\section{ACKNOWLEDGEMENTS}

The Australia Telescope Compact Array (ATCA) is part of the Australia Telescope National Facility which is funded by the Commonwealth of Australia for operation as a National Facility managed by Australian Commonwealth Scientific and Industrial Research Organisation (CSIRO). This paper includes archived data obtained through the Australia Telescope Online Archive (http://atoa.atnf.csiro.au). We used the KARMA and MIRIAD software packages developed by the Australia Telescope National Facility (ATNF) and the PYTHON programming language. This work is part of the project 176005 'Emission nebulae: structure and evolution' supported by the Ministry of Education, Science, and Technological Development of the Republic of Serbia.

\section{REFERENCES}

Aharonian F. et al., 2007, A\&A, 464, 235

Bell A. R., Matthews J. H., Blundell K. M., 2019, MNRAS, 488, 2466

Bell A. R., Schure K. M., Reville B., 2011, MNRAS, 418, 1208

Borkowski K. J., Gwynne P., Reynolds S. P., Green D. A., Hwang U., Petre R., Willett R., 2017, ApJ, 837, L7

Borkowski K. J., Reynolds S. P., Green D. A., Hwang U., Petre R., Krishnamurthy K., Willett R., 2010, ApJ, 724, L161

Borkowski K. J., Reynolds S. P., Hwang U., Green D. A., Petre R., Krishnamurthy K., Willett R., 2013, ApJ, 771, L9

Bozzetto L. M., Filipović M. D., Urošević D., Kothes R., Crawford E. J., 2014, MNRAS, 440, 3220

Bozzetto L. M. et al., 2017, ApJS, 230, 2

Braiding C. et al., 2018, PASA, 35, e029

Brose R., Sushch I., Pohl M., Luken K. J., Filipovic M. D., Lin R., 2019, A\&A, 627, A166

Burton M. G. et al., 2013, PASA, 30, e044

Cappellaro E., Barbon R., Turatto M., 2005, in Marcaide J.-M., Weiler K. W., eds, IAU Colloq. 192: Cosmic Explosions, On the 10th Anniversary of SN1993J, Vol. 99. Springer, Berlin, Heidelberg, p. 347

Caprioli D., Spitkovsky A., 2014, ApJ, 783, 91

Carlton A. K., Borkowski K. J., Reynolds S. P., Hwang U., Petre R., Green D. A., Krishnamurthy K., Willett R., 2011, ApJ, 737, L22

Cherenkov Telescope Array Consortium et al., 2019, Science with the Cherenkov Telescope Array. World Scientific Publishing Co., Singapore Chevalier R. A., 1976, ApJ, 207, 872

Cohen R. J., 1975, MNRAS, 171, 659

de Grijs R., Bono G., 2016, ApJS, 227, 5

De Horta A. Y. et al., 2014, Serb. Astron. J., 189, 41 
Dwarkadas V. V., 2005, ApJ, 630, 892

Farnes J. S., 2012, PhD thesis, University of Cambridge

Francis C., Anderson E., 2014, MNRAS, 441, 1105

Fukui Y. et al., 2012, ApJ, 746, 82

Gooch R., 2011, Astrophysics Source Code Library, record ascl:1102.018

Gray A. D., 1994, MNRAS, 270, 847

Green D. A., Gull S. F., 1984, Nature, 312, 527

Green D. A., Reynolds S. P., Borkowski K. J., Hwang U., Harrus I., Petre R., 2008, MNRAS, 387, L54

Gómez Y., Rodríguez L. F., 2009, RMxAA, 45, 91

Gull S. F., 1973, MNRAS, 161, 47

Gull S. F., 1975, MNRAS, 171, 263

Han J. L., Manchester R. N., van Straten W., Demorest P., 2018, ApJS, 234, 11

Harvey-Smith L., Gaensler B. M., Kothes R., Townsend R., Heald G. H., Ng C.-Y., Green A. J., 2010, ApJ, 712, 1157

H.E.S.S. Collaboration et al., 2018, A\&A, 612, A6

Hunter J. D., 2007, Comput. Science \& Engineering, 9, 90

Hurley-Walker N. , 2019, Publications of the Astronomical Society of Australia, 36, E047

Hurley-Walker N. et al., 2017, MNRAS, 464, 1146

Jiang Z. J., Zhang L., Fang J., 2013, MNRAS, 433, 1271

Johnston S., McClure-Griffiths N. M., Koribalski B., 2004, MNRAS, 348, L19

Kerr F. J., Lynden-Bell D., 1986, MNRAS, 221, 1023

LaRosa T. N., Kassim N. E., Lazio T. J. W., Hyman S. D., 2000, AJ, 119, 207

Maggi P. et al., 2019, A\&A, 631, A127

Manchester R. N., Hobbs G. B., Teoh A., Hobbs M., 2005, AJ, 129, 1993

Maxted N. et al., 2013, PASA, 30, e055

Milne D. K., 1987, Aust. J. Phys., 40, 771

Murphy T., Gaensler B. M., Chatterjee S., 2008, MNRAS, 389, L23
Nord M. E., Lazio T. J. W., Kassim N. E., Hyman S. D., LaRosa T. N., Brogan C. L., Duric N., 2004, AJ, 128, 1646

Pavlović M. Z., 2017, MNRAS, 468, 1616

Reynolds S. P., Borkowski K. J., Green D. A., Hwang U., Harrus I., Petre R., 2008, ApJ, 680, L41

Reynolds S. P., Borkowski K. J., Green D. A., Hwang U., Harrus I., Petre R., 2009, ApJ, 695, L149

Reynolds S. P., Gaensler B. M., Bocchino F., 2012, Space Sci. Rev., 166, 231

Roper Q. et al., 2018, MNRAS, 479, 1800

Roy S., Pal S., 2014, in Ray A., McCray R. A., eds, IAU Symp. Vol. 296, Supernova Environmental Impacts, Kolkata (Calcutta), p. 197

Sano H. et al., 2010, ApJ, 724, 59

Sano H. et al., 2015, ApJ, 799, 175

Sano H. et al., 2019, ApJ, 873, 40

Sault R. J., Teuben P. J., Wright M. C., 1995, in Astronomical Data Analysis Software and Systems IV. p. 433

Telezhinsky I., Dwarkadas V. V., Pohl M., 2013, A\&A, 552, A102

Uchiyama Y., Aharonian F. A., Tanaka T., Takahashi T., Maeda Y., 2007, Nature, 449, 576

Urošević D., 2014, Ap\&SS, 354, 541

Urošević D., Pavlović M. Z., Arbutina B., 2018, ApJ, 855, 59

van den Bergh S., Tammann G. A., 1991, ARA\&A, 29, 363

Völk H. J., Berezhko E. G., Ksenofontov L. T., 2003, A\&A, 409, 563

Wayth R. B. et al., 2015, PASA, 32, e025

Wilson W. E. et al., 2011, MNRAS, 416, 832

Xu J., Han J.-L., 2014, Res. Astron. Astrophys., 14, 942

Yao J. M., Manchester R. N., Wang N., 2017, ApJ, 835, 29

This paper has been typeset from a $\mathrm{T}_{\mathrm{E}} \mathrm{X} / \mathrm{LT} \mathrm{E} \mathrm{X}$ file prepared by the author. 